\title{
Continuous-time (Ross-type) portfolio separation, (almost) without Itô calculus
}

\author{
Nils Chr. Framstad (D) \\ Department of Economics, University of Oslo, Oslo, Norway
}

\begin{abstract}
This paper shows how the distributions-based portfolio separation theorem - also known as the mutual fund theorem - for elliptical and stable distributions carries over from a static to a continuoustime model. Without invoking Itô stochastic calculus, only the definition of the Itô integral, we generalize and simplify an approach of Khanna and Kulldorff (http://link.springer.com/article/ 10.1007\%2Fs007800050056 Finance Stoch. 3 (1999), pp. 167-185). In addition to (re-) covering the classical cases, this paper also gives separation results for non-symmetric stable distributions under no shorting-conditions, including a new case of one fund separation without risk-free opportunity. Applicability of the skewed cases to insurance and banking is discussed, as well as limitations.
\end{abstract}

\section{ARTICLE HISTORY}

Received 20 August 2015

Accepted 11 December 2015

\section{KEYWORDS}

Portfolio separation; mutual fund theorem; elliptical distributions; (Lévy-Pareto) $\alpha$-stable distributions; Lévy processes; stochastic dominance; portfolio constraints; incomplete markets; risk management

\section{AMS SUBJECT} CLASSIFICATIONS 91G10; 91G80; 60E07; $60 \mathrm{E} 15 ; 60 \mathrm{G} 50 ; 60 \mathrm{G} 51$; $60 \mathrm{G} 52 ; 60 \mathrm{H} 05 ; 93 \mathrm{E} 20 ; 49 \mathrm{~K} 45$

\section{Introduction}

The concept of portfolio separation can be briefly formulated as follows: under what conditions can a market of a large number of investment opportunities be replaced by a few market indices («funds») without the agents being worse off? The prototypical result is two-fund separation (Tobin, [62]) which is the property that two market indices suffice; it is frequently referred to as two-fund monetary separation if one can choose one of them as a risk-free investment opportunity, i.e. with certain return. Tobin's work builds on the mean-variance approach by Markowitz [34]; as a historical note, Markowitz was predated by more than a decade by de Finetti [11] (see Barone [63,64] for a detailed review and translation; see also Pressaco and Serafini [50] and Markowitz' account [35] where he also credits Roy [54]). Tobin obtained the result in a single-period model with either Gaussian returns or quadratic utility, and since then, the results on portfolio separation have mainly fallen into these categories:

(i) Characterization of the agents who - for «any») market - are satisfied with a fixed set of funds, irrespective of their wealth. Separation in this sense is a property of a given preference ordering - typically, a utility function for expected utility. The funds will be common to those agents who share this utility function, so that changes in wealth

CONTACT Nils Chr. Framstad n.c.framstad+research@econ.uio.no 
due to profits and losses will not change the funds, only the allocation among them - this assuming that preferences and parameters are invariant over time.

A characterization of those utility functions which admit two-fund separation for a single-period model, was given in 1970 by Cass and Stiglitz [9]. Cass-Stiglitz type portfolio separation is still a topic of research, see e.g. Schachermayer et al. [58] in a continuous-time model using modern probabilistic methods, or Guasoni and Robertson [23].

(2) Characterization of the returns distributions for which «any») agent under consideration will be satisfied with the same few funds.

A characterization of those returns distributions which yield $k$-fund separation over expected utility maximizers in a single-period model, was given in 1978 by Ross [53] in terms of stochastic dominance. His main focus was risk-averse agents, i.e., a second-order stochastic dominance criterion; the version valid for all greedy agents (the focus of this paper, a first-order stochastic dominance criterion) appears in footnote remarks. As it is not trivial which distributions admit the construction, this Ross-type portfolio separation is still a topic for research; this author's recent contribution [19] does in essence boil down to the work of verifying the Ross criteria.

Ross-type two-fund separation is the property used to establish the Capital Asset Pricing Model. Separation properties also follow from other types of models or assumptions, e.g. through the application of risk measures, as pointed out by this author [17] and later independently by De Giorgi et al. [22], while He and Zhou [27] approach by way of quantiles.

\subsection{Ross-type portfolio separation, and this paper's contribution}

This paper is about the distributions-based portfolio separation property, but in continuous time. The setup is a consumption-portfolio choice problem where there are either insurance profits/losses modelled by Levy processes - or there are traded opportunities, whose prices are geometric processes with Levy processes as driving noise. The market is free from transaction costs, although certain constraints on the portfolios will be a main focus of interest. We derive conditions for portfolio separation valid for any agent whose preferences are consistent with first-order stochastic dominance, and who can satisfy the portfolio constraints.

The continuous-time separation properties will be derived from the analogous singleperiod property. The large body of literature on the single-period model is therefore most relevant. Omitting a few fascinating details where insightful assertions have turned out to admit counterexamples, we merely mention a few contributions: Feldstein [16], Borch [6], Agnew [1], Samuelson [56,57] and Fama [14]; the latter two establish the result for (matrixtransformed) iid $\alpha$-stable distributions. Following Ross [53], Owen and Rabinovitch [49] showed that any elliptically distributed vector would satisfy the Ross conditions, while about simultaneously, Chamberlain [10] established that under square integrability, ruling out non-Gaussian $\alpha$-stables, these would be precisely those distributions for which every risk-averse agent would be a mean-variance-optimizer. This ties the knot back to the original mean-variance approach; indeed, the key properties were to be found as far back as Schoenberg $[59,60]$ in 1938 , before modern portfolio theory. 
In continuous time, two-fund separation in a complete geometric Brownian market was obtained by Merton [38] (see Section 5 for the distributions-type theorem: corrections to other parts appear in [39] and [61]) by means of dynamic programming, which has prevailed as the standard tool. Fast-forwarding to 1999, Khanna and Kulldorff [31] employed a technique which cleverly fits a Ross-type reasoning more directly to continuoustime models, offering a simple method to remove the risk aversion assumption and the (irrelevant!) completeness assumption from the Merton approach. Their method appears informal, but is arguably sufficiently rigorous given knowledge of the basic properties of Brownian motion, and captures in an easy way the case of «no short positions» constraints. They do however assume geometric Brownian prices and the existence of a risk-free investment opportunity.

This paper removes or generalizes many of the assumptions made in the Khanna and Kulldorff [31] paper, in mainly three directions:

- Probability laws. As in the single period case, we can replace the Gaussian risk by members of a larger distribution class. When formulated on differential form - but not when considering log-returns - we can formally replace the driving Brownian motion by an elliptically distributed Lévy process and obtain the same result, just as in the single period case. And just like for a single period, there are other symmetry properties that admit separation. Under those portfolio constraints considered by [31], we cover more general distributions than the subclass of symmetric $\alpha$-stable considered in the classical works [14] and [56], and in post-y2k works as [7], [46] and [47]. In particular, the non-symmetric cases we cover, are rarely if ever treated in the portfolio literature.

- Portfolio constraints. For elliptical distributions we allow for linear constraints, thus covering the classical case where no risk-free opportunity exists. Also, the result can be adapted to cover different lending and borrowing rates. Those results fail to hold for non-elliptical separating distributions, see [20], although an exceptional case will appear in Corollary 5.2, which to the best of the author's knowledge is unpublished even for the single-period model. Furthermore, the radial constraints that [31] employ, facilitate also certain non-symmetric distributions.

- Preferences. In [31], time-additive expected utility is assumed. This assumption is unnecessarily strong, and we weaken it to assume that agents partially order probability distributions consistently with a dynamic version of first-order stochastic dominance. We can still - like in [31] - drop the preference of more to less if there is free disposal of wealth, and instead assume that only probability distributions are ordered: the restriction to the span of the fund(s) will not remove anything from the opportunity set.

Example 3.5 gives an example of preferences not covered by [31], but where this paper can conclude that the geometric Brownian Black-Scholes model (as [31] employs), leads to the agent adapting somewhere on the Merton line.

First-order stochastic dominance does correspond to agreement across all every expected increasing utility maximizers, but it also covers preferences that are far from any von Neumann-Morgenstern expected utility representation. For example, univariate Gaussians are ordered only if they have the same variance: consider therefore an arbitrary partial ordering of variances (i.e. of the nonnegative reals), where for equal variances, ties 
are broken by picking the largest mean. Our approach will apply to this kind of preferences. Our model has however one thing firmly in common with vNM theory: homogeneous beliefs, in that everybody agrees on probabilities.

\subsection{Why not dynamic programming or a Pontryagin maximum principle?}

The method used in this paper - as well as by [31] - distinguishes itself from dynamic programming as well as from the perturbation arguments that lead to different guises of the Pontryagin maximum principle. Both of these methods employ some kind of tradeoff between present and future, in order to form conditions for an optimal or $\epsilon$-optimal choice for today. In the Pontryagin principle, the trade-off enters by a shadow prices on state changes; in dynamic programming, one typically uses even stronger assumptions, as one invokes the value of the programme as a function of state, implicitly assuming the existence of a value function and some kind of Markov property. In contrast, this paper makes no assumption of intertemporal substitutability - indeed, the assumption of such in [31] is redundant. Under the distributional assumptions, the separation theorem can be obtained by exploiting only those improvements that can be made instantaneously without affecting the wealth and consumption flow of the future, other than by exchanging to a stochastically conditionally independent copy.

To clarify the paper's title, which promises an exposition with hardly any Itô calculus, we shall employ the Itô integral as the limit in probability of step integrals. We shall do without the calculus that involves the second-order term of the Itô change of variables rule, which in textbook expositions like e.g. [40] or [45] enters in the second-derivative term(s) in the Hamilton-Jacobi-Bellman equation - also, we avoid the change of variable rule for the non-Brownian jump processes. We shall (cf. formula (1)) alter the «consumption» choice process in order to avoid the change of variable - which in Itô calculus would not involve any second-order term as long as we stick to the standard assumption of locally risk-free numéraire. At one point we will nevertheless allow an auxilliary market state $M(t)$ to evolve in a strongly Markovian way, though state-dependent, and thus we allow it to be represented in terms of a stochastic differential Equation (10).

\subsection{Organization of the paper, and notation}

The next subsection will give and justify the model of wealth accumulation - it is essentially textbook material, though with a few quirks due to the generality that allows jumps past zero in certain cases. Section 2 will review the concepts of first-order stochastic dominance, then motivate which of them to use in the dynamic setup. Section 3 will then give a model with full assumptions, and the main argument to be applied in the subsequent two sections; Sections 4 and 5 will establish the separation results for the elliptical laws, resp. the $\alpha$-stable laws. Section 6 will discuss the differences between these distribution classes' separation properties, discuss applicability and limitations of the new results obtained in the paper, and conclude. Proofs are collected in Appendix 1.

The paper will employ the following notation and terminology: The differential « $\mathrm{d}$ » like in $(\star)$ always denotes the Itô (i.e. non-anticipative) type integral, as one would expect in stochastic finance. $\triangle$ denotes discrete non-anticipative time-increment. The symbol $\sim$ will 
denote equality in law or finite-dimensional distributions - in particular, for uni-/multivariate random vectors, the distribution itself. Matrices will be denoted with slanted capital Greek letters. $\Xi^{1 / 2}$ denotes the positive semidefinite square root of a positive semidefinite $\Xi$. Superscript ${ }^{\top}$ denotes transpose. Vectors - by default columns - will be denoted in boldface ( 1 will be the vector of ones), and only random vectors and vector-valued stochastic processes will be boldface Latin capitals - however, the portfolio $v$ which can be controlled at any time, in minuscle. The reader can note the agent can not influence anything in Greek letters unless specified as a function of choice variables. We shall use $X$ or $\boldsymbol{X}$ for the generic random variable - not for processes although those may be allowed to be denoted with or without the time argument - and $x$ or $\boldsymbol{x}$ for the generic free variable for e.g. static optimization. A random vector $\boldsymbol{X}$ is symmetric iff $-\boldsymbol{X} \sim \boldsymbol{X}$. P and E will denote probability resp. expectation. Sets will be denoted in blackboard bold, with $\mathbb{R}_{+}^{n}$ denoting the closed positive orthant. $\mathbb{A}$ is radial if $\forall a>0, a \boldsymbol{x} \in \mathbb{A}$ iff $\boldsymbol{x} \in \mathbb{A}$. Sigmaalgebras on the probability space are denoted in fraktur letters, where the $\vee$ symbol - e.g. $\mathfrak{S} \vee \mathfrak{F}$ - denotes the smallest majorizing sigmaalgebra. Filtrations are denoted in boldface fraktur. The letters $\mathfrak{S}, \mathfrak{H}_{t}, \mathfrak{H}$ will have special meanings: $\mathfrak{S}^{X}$ will denote the sigmaalgebra generated by a single random $\boldsymbol{X}$; we shall allow families of sets/events as superscripts as well as random variables. For a process, e.g. $Y$ we shall denote by $\mathfrak{H}_{t}^{Y}$ (the letter a mnemoniac for history) the sigma-algebra $\vee_{t^{\prime} \leq t} \mathfrak{S}^{Y\left(t^{\prime}\right)}$, while the $\mathfrak{H}^{Y}$ (boldface, without subscript $t$ ) will denote the filtration, i.e. the entire increasing family $\left\{\mathfrak{H}_{t}^{Y}\right\}_{t}$ - although all sigmaalgebras will be assumed complete(d) in the Cauchy sense, cf. Assumption 3.2(a). For each filtration

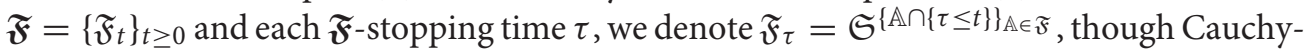
completed.

Finally, «measurable» shall mean the information sense of measurability with respect to sigma-algebras mentioned in the previous paragraph. Borel (taken to mean completed Borel) measurability will be taken for granted as long as there is nothing to prove, and in the very few cases left, we shall speak of «Borel» functions rather than using the «measurable» term.

\subsection{The wealth dynamics - non-traded and traded opportunities}

The model for portfolio value («wealth») Y shall be assumed a controlled stochastic (Itô) process of the form

$$
\mathrm{d} Y(t)=\boldsymbol{v}\left(t^{-}\right)^{\top}[\boldsymbol{\mu} \mathrm{d} t+\Gamma \mathrm{d} \boldsymbol{Z}(t)]-\mathrm{d} C(t)-\mathrm{d} D \quad \text { for } t \geq 0
$$

with a given history and present $\{(Y(t), C(t))\}_{t \leq 0}$, and $D(t)=0$ for $t \leq 0$.

- take for the moment the vector $\boldsymbol{\mu}$ and the matrix $\Gamma$ to be constant, although this will be generalized. Throughout the paper, $\boldsymbol{Z}$ will be a Lévy process; for a reference, see Applebaum's book [2]. The form ( $\star$ ), with $\mathrm{d} t$-absolutely continuous drift, fits a continuous time framework, although the results are easy to fit to discrete time or a combination. The disposal $D$ - assumed nondecreasing, allowing the agent to throw away wealth without consuming it - facilitates even non-greedy agents, but is introduced mainly to simplify proofs.

The model ( $\star$ ) will be assumed, and the following non-rigorous discussion serves merely as a motivation and to clarify a few deviations from the textbook model by allowing a $\boldsymbol{Z}$ 
that could cause $Y$ to jump across zero - we model absolute returns and not logreturns. The arguably simplest model leading to $(\star)$, is when holding one unit of each («risky») investment opportunity number $i$, incurs a profit/loss flow assumed to include all costs, like funding costs for e.g. statutory risk capital - assumed linear in the exposure $v_{i}$. The profits/loss process can then be modelled as $\boldsymbol{\mu} \mathrm{d} t+\Gamma \mathrm{d} \boldsymbol{Z}$ - unlike for traded opportunities, there are no price movements; if there are, they could be introduced with the traded model below. Thus we only need to redefine consumption to arrive at $(\star)$; we define $C$ through

$$
\mathrm{d} C=\mathrm{d} \breve{C}-\rho Y \mathrm{~d} t, \quad \text { where } \breve{C} \text { is actual consumption }
$$

and where $\rho$ is the risk-free interest rate on the endowment; for the moment we assume it to exist, only to forbid investment in it in the case where it does not. We shall compare strategies which yield same probability law for $Y$, and given this, orderings of actual consumption processes $\breve{C}$ will be the same as orderings of $C$ processes. This simple argument makes $(\star)$ valid also for e.g. non-traded insurance portfolios, as long as everything is linear in exposure.

For the textbook model for traded investment opportunities at price processes $P_{i}=P_{i}(t)$, let $u_{i}$ units held at time $t$, at market value $u_{i} P_{i}$, one assumes the self-financing property, defined by the ansatz that the value of the position evolves as $u_{i}(t) \mathrm{d} P_{i}$ (where $u$ is predictable, i.e. $u_{i}(t)=u_{i}\left(t^{-}\right)$if one-sided continuous). With the canonical form $\mathrm{d} P_{i}(t)=P_{i}\left(t^{-}\right)\left(\rho \mathrm{d} t+\mathrm{d} G_{i}\right)$ for price fluctations - modelling excess returns over riskfree interest, for the moment assumed to exist - the market value of the position obeys (post-discounting) $v_{i} \mathrm{~d} G_{i}$ where $v_{i}=u_{i} P_{i}=$ the value exposed. Again, define $\mathrm{d} C$ as the differential of actual consumption less $\rho Y \mathrm{~d} t$, we are done with the formal exercise.

Remark 1.1: Limited liability is not assumed, unless implied by the distributional properties of the $G_{i}$; conditions are given in Remark 4.2. Let us briefly discuss the situation of jump past zero - say, a possible jump from price $p$ to price $-p$. Even if this possibility is justified, it is arguably still questionable that the geometric model assumes an equal intensity to jump back from $-p$. The model can however be interpreted such that jumps to $\leq 0$ (and, as a possible modeling choice, some or all of the other jumps as well!) have to be settled in cash immediately like in the non-traded model. Note that it is then crucial that the investment opportunity does not disappear - it should be immediately available with the same dynamics, otherwise the stochastic dependence between wealth jumps and the future opportunity set will easily destroy separation properties (even for power utility, cf. [18]). That in particular goes for jumps to zero; for example, one could use such a model for a catastrophe-linked bond, which pays coupon at a certain spread, but which defaults to zero at pre-specified Poisson jump events. For our model to work in this case, a new bond - at the same dynamics - must then be immediately available to investors.

\section{Stochastic dominance and the Ross argument}

This section will review first-order stochastic dominance for random variables and point out what is the natural generalization to stochastic processes, for the purposes of this paper. Recall that a real-valued random variable $X^{*}$ first-order (weakly) stochastically dominates another variable $X$, if any of the three following equivalent criteria hold:

(i) there exists some nonnegative random variable $X_{+}$such that $X \sim X^{*}-X_{+}$. 
(ii) $\mathrm{E}[f(X)] \leq \mathrm{E}\left[f\left(X^{*}\right)\right]$ for every bounded nondecreasing function $f$.

(iii) $\mathrm{CDF}_{X} \geq \mathrm{CDF}_{X^{*}}$

An ordering of probability distributions «preferring more to less» should rank $X^{*}$ at least as good as $X$. Conversely, if neither of $X$ and $X^{*}$ first-order dominates the other, then there exist two expected utility maximizers which rank them oppositely - indeed, by (iii) there are numbers $\bar{x}_{1}$ and $\bar{x}_{2}$ such that $f_{1}(x):=1_{\left\{x \geq \bar{x}_{1}\right\}}$ and $f_{2}(x):=1_{\left\{x \geq \bar{x}_{2}\right\}}$ disagree on the orderings.

The equivalence between (i)- and (ii)-type criteria holds far beyond real-valued variables. It is established for partially ordered Polish spaces (Kamae and coauthors $[29,30]$ ), covering our dynamic model. It shall turn out most convenient to apply the concept (i) - also known as the mass transfer definition. The next subsection reformulates the static model with one trading decision, into a two-period model in a way that naturally generalizes to several periods and to continuous time.

Remark 2.1: The term «Ross-type» separation is justified by the concept that we consider separation a property of probability distributions valid for «all» agents in a wide class despite the main focus of Ross [53] being second-order stochastic dominance, restricting to risk-averse agents and leaving the rest to footnotes. For second-order dominance, the common analogues to i) resp. (ii) are to assume $X_{+}$to have nonnegative expectation, resp. to require to hold merely for the $f$ which are nondecreasing and concave. Both cases involve integrability assumptions we do not want to impose in this paper. But even if we disregard integrability issues, adapting the second-order criterion to the dynamic framework seems not straightforward; there seems to be no analogue to [30]. A few references on the matter are summarized in [48, footnotes 3-6]. For this reason, this paper does not consider riskaversion apart from informal remarks.

\subsection{Heuristics on dominance and separation in a two-period model}

This subsection will review how stochastic dominance leads to separation in what is essentially a single-period model reformulated to generalize to a dynamic model. We do not specify in full detail, leaving the assumptions needed for proper theorems for Section 3. For the purpose of the illustration, the reader can consider multinormal returns. For a single period model, take constant coefficients in $(\star)$ and restrict to times 0 and 1: portfolio has to be chosen (at $t=0$ ) constant over the period. Restrict $C$ to being nondecreasing. Consider the following examples in order of complexity,

- Single period, agent cares only about $Y(1)$ :

Nothing will be consumed. Suppose that for two portfolios $v^{*}$ and $v$ we have

$$
\boldsymbol{v}^{* \top} \Gamma[\boldsymbol{Z}(1)-\boldsymbol{Z}(0)] \sim \boldsymbol{v}^{\top} \Gamma[\boldsymbol{Z}(1)-\boldsymbol{Z}(0)] \quad \text { and } \quad \boldsymbol{v}^{* \top} \boldsymbol{\mu} \geq \boldsymbol{v}^{\top} \boldsymbol{\mu} .
$$

Then the portfolio return using $v^{*}$ first-order stochastically dominates the portfolio return using $v-$ this exhibited by taking $X_{+}=\left(\boldsymbol{v}^{*}-\boldsymbol{v}\right)^{\top} \boldsymbol{\mu}$. When initial wealth, common to the two strategies, is added, then final wealth $Y^{*}(1)$ stochastically dominates $Y(1)$. Thus any greedy agent should prefer using $\boldsymbol{v}^{*}$ to using $\boldsymbol{v}$. With $\boldsymbol{Z}$ Gaussian, the agent can improve over $v$ by choosing one with same portfolio variance 
$\boldsymbol{v}^{* \top} \Gamma \Gamma^{\top} \boldsymbol{v}^{*}=\boldsymbol{v}^{\top} \Gamma \Gamma^{\top} \boldsymbol{v}$, but higher mean - unless, of course, $\boldsymbol{v}$ already maximizes mean for given variance.

- Single period, «for equal final wealth, prefer more final consumption».

We make this more precise, by considering preferences at time 1 and time 0 . At time 1, the agent will for given post-consumption wealth $Y\left(1^{+}\right)$, want to consume as much as possible. Say, if in one case the agent has $Y(1)=56$ and in another she has $Y^{*}(1)=58$, and in the former wishes to consume 17 , then in order to make them comparable, we have to consume 19 in the latter case. At time 0 , we cannot order statewise, as we do not know $\tilde{Z}$, so instead we argue for the «starred» strategy of the previous bullet point by the following argument: Let consumption $C\left(1^{+}\right)-C\left(1^{-}\right)$at time 1 be a function $c$ of $Y(1)$, since everything is equal between the strategies. For the starred strategy, however, choose $c^{*}\left(Y^{*}(1)\right)=c\left(Y^{*}(1)\right)+\left(\boldsymbol{v}^{*}-\boldsymbol{v}\right)^{\top} \boldsymbol{\mu}$. In joint distribution viewed from time zero, this is preferable, because $\left(\boldsymbol{v}^{*}-\boldsymbol{v}\right)^{\top} \boldsymbol{\mu} \geq 0$ :

$$
\left(Y^{*}\left(1^{+}\right), c^{*}\left(Y^{*}(1)\right)\right) \sim\left(Y\left(1^{+}\right), c(Y(1))+\left(0,\left(\boldsymbol{v}^{*}-\boldsymbol{v}\right)^{\top} \boldsymbol{\mu}\right)\right.
$$

Note that in the next section, we shall require equal (in law) intermediate wealth as well. The next bullet point will indicate two ways to deal with this.

- Consumption before time 1 matters as well:

If intermediate consumption does not matter, but consumption at time 0 does, then the starred strategy can just mimic at time zero, and the rest goes as the previous bullet point. However if intermediate consumption matters, we must be careful: suppose we observe intermediate wealth as well. Consumption $C\left(T^{+}\right)$up to time $T^{+}$, will then depend through some transformation $F_{T}(Y(\cdot))$ on wealth up to and including time $T$. If we try to plug $Y^{*}$ into this $F$, we make a certain error, as it accumulates (in law) faster. We can resolve this by gradually consume the excess, and in Lemma 3.11 we shall establish this construction through the limit of short time periods. Here is an intuitive solution though: dispose of wealth, gradually, at rate $\left(\boldsymbol{v}^{*}-\boldsymbol{v}\right)^{\top} \boldsymbol{\mu}$. Plug the resulting wealth, with equal law, into $F$ to determine consumption. This will not make a greedy agent better off than the «unstarred» strategy, but no worse either.

The second bullet point exhibits the mass transfer concept as the one to apply in the dynamic setting. The third bullet point exhibits the method we will use to construct an improvement in continuous time, through approximation by discrete periods. It also exhibits free disposal as a handy tool. We shall split into cases where free disposal is possible, and where it is not; at one stage, the proof of Theorem 3.12 will actually first make, and then lift, an assumption of free disposal.

\section{Standing assumptions and definitions for the dynamic model, and the main argument}

The modelling framework will take as starting point the usual filtered probability space, in Assumption 3.2 below. Unlike the common portfolio optimization approach, we shall allow the agent to draw independent randomization in the sense that the agent can observe the common filtration and in addition draw a lottery independent of everything else. This is arguably uncommon in the literature on portfolio choice, wherein such an opportunity 
would not be employed; in our setting, however, we need to ensure that we keep the opportunity set even when restricting the portfolio to the span of the funds.

By abuse of logic, let us restate the model before we define the space on which it is defined:

Definition 3.1: The following is the model for the agent's wealth $Y$ (real-valued process) in terms of the strategy $(C, v)$ consisting of a real-valued (cumulative) consumption process $C$, (cumulative) disposal $D$, and a portfolio process $\boldsymbol{v}$, the latter taking values in a control region $\mathbb{V}=\mathbb{V}_{t} \subseteq \mathbb{R}^{n}$, henceforth also referred to as a portfolio constraint; the unconstrained case will refer to when $\mathbb{V}=\mathbb{R}^{n}$.

$$
\begin{aligned}
& \mathrm{d} Y(t)=\boldsymbol{v}\left(t^{-}\right)^{\top}[\boldsymbol{\mu} \mathrm{d} t+\Gamma \mathrm{d} \boldsymbol{Z}(t)]-\mathrm{d} C(t)-\mathrm{d} D(t) \quad \text { for } t \geq 0 \\
& \text { with }\{Y(t), C(t)\}_{t \leq 0} \text { given and } D=0 \text { for } t \leq 0 .
\end{aligned}
$$

In both cases, $\boldsymbol{Z}$ is the driving noise, $\boldsymbol{\mu}$ and $\Gamma$ are the market coefficients.

The assumption that the past is the entire negative half-line, is without loss of generality, as we can extrapolate with a constant. We now state the assumptions for this model.

Assumption 3.2: (for the probability space) The following are standing - unless otherwise specified - assumptions for the probability space we define the model ( $\star$ ) on; furthermore, some notation and terminology is introduced.

(a) Assume as given a Cauchy-completed filtered probability space $(\Omega, \mathfrak{F}, \mathfrak{F}, \mathrm{P})$ with $\mathfrak{F}$ right-continuous. Every $\mathfrak{F}_{t}$ and every sigma-algebra defined in the following, will without mention be assumed contained in $\mathfrak{F}$, and completed by augmenting with the nullsets of $\mathfrak{F}$ - by assumption including all their subsets, and also all history up to time 0 .

(This means that $(\Omega, \mathfrak{F}, \mathfrak{F}, \mathrm{P})$ satisfies the so-called usual conditions, and furthermore that the agent at time 0 knows her past, as it is taken as non-random; Definition 3.6 will ensure that the past is known at any stopping time.)

(b) On this probability space there is an adapted strongly Markov process pair $(\boldsymbol{Z}, M)$ with sample paths a.s. right-continuous with left limits, and such that $\mathbf{Z}$ is a Lévy process in $\mathbb{R}^{m}$. Except when formula (11) dictates otherwise, $\boldsymbol{Z}$ is assumed decomposable into the sum of a Lévy process with symmetrically distributed increments (i.e. a limit of symmetric martingales of bounded jumps), and a martingale.

Unless otherwise stated, $M(t)=t$-i.e., deterministic. In order to state such a deviation, $M$ will be referred to as a «market state» variable (that affects the model parameters); we do for simplicity only consider the case of one-dimensional $M$, leaving it to the reader to verify the generalization in question.

(c) Each agent is permitted to do any $(\boldsymbol{Z}, M)$-independent randomization of their choice. We will invoke this in the arguments whenever necessary symbolically denote it as a process $\boldsymbol{L}$, assumed predictable.

(d) The market coefficients $\boldsymbol{\mu}$ (taking values in $\mathbb{R}^{n}$ ) and $\Gamma$ (taking values in $\mathbb{R}^{n \times m}$ ), are functions of only $\left(t, M\left(t^{-}\right)\right)$- and constant unless otherwise specified.

(e) The given control region $\mathbb{V} \subseteq \mathbb{R}^{n}$ is a closed and unless otherwise specified, fixed set; the most significant deviation is specified by no risk-free opportunity when $\mathbb{V}=\mathbb{V}_{t}$ is restricted to the agent-specific set $\left\{\boldsymbol{v} ; \boldsymbol{v}^{\top} \mathbf{1}=Y\right\}$ or a subset thereof. In any case, $\mathbb{V}_{t}$ can for each agent be written as $\left\{\boldsymbol{x} ; f\left(t, \boldsymbol{x},\left\{Y_{t-s}\right\}_{s>0}\right) \geq 0\right\}$ where $f$ is a nonrandom 
real functional, satisfying the following: whenever $Y\left(T^{+}\right)=Y\left(T^{-}\right)$then for each $\epsilon>0$ and $\boldsymbol{x} \in \mathbb{V}_{T}$ there exists a stopping time $\tau>T$ a.s. such that the distance from $\boldsymbol{x}$ to $\mathbb{V}_{t}$ is $<\epsilon$ for $t \in(T, \tau)$.

(f) Consider any triplet $\boldsymbol{v}, \tau, \hat{\tau}$ with $\hat{\tau} \geq \tau$ being stopping times and $\boldsymbol{v}$ being bounded, predictable and taking values in $\mathbb{V}$, such that on the event $\{\tau<\infty\}$ we have $\hat{\tau}-\tau$ being $\mathfrak{F}_{\tau}$-conditionally bounded and not a.s. zero. Then the variable $\int_{\tau}^{\hat{\tau}} \boldsymbol{v}^{\top}[\boldsymbol{\mu} \mathrm{d} t+\Gamma \mathrm{d} \boldsymbol{Z}]$ is not a.s. nonnegative except if $\boldsymbol{v}$ vanishes almost everywhere on $(\tau, T)$, and neither is it a.s. nonpositive if $\mathbb{V} \subseteq\left\{\boldsymbol{v} ; \boldsymbol{v}^{\top} \mathbf{1}=Y\right\}$; in the latter case, assume unless otherwise specified that $\max _{i} \mu_{i} \geq 0 \geq \min _{i} \mu_{i}$. This is without loss of generality, by adjusting the fictitious interest rate $\rho$. In particular, by this adjustment, we can assume that $\boldsymbol{\mu}$ is not a non-zero scaling of $\mathbf{1}$; that means in particular, an expected return common to all opportunities, is transformed away.

Remark 3.3: The decomposition of $\boldsymbol{Z}$ in item (b) generalizes the martingale property by allowing symmetry to generalize zero mean. Only in Section 5 we shall generalize further.

The randomization variable $\boldsymbol{L}$ of item (c) is a departure from the common setup, and presents technical nuissances if pre-specified in a way that can cover all thinkable choices of randomization; at worst, sample paths would no longer need to be Borel. We shall deal with this by comparing any admissible strategy (as to be defined in Definitions 3.6) pairwise with one that is also admissible (meaning, intuitively, at least equally well-behaved).

Item (f) is connected to absence of arbitrage opportunities, but has some other consequences too; it ensures that the case we refer to as «no risk-free opportunity» does indeed not admit any such, and that there is only one in the case with risk-free opportunity. The assumption is not sufficient to rule out free lunches by unbounded strategies, but this paper need not rule these out; see the admissibility concept in Definition 3.6.

The agent has preferences over the distribution over wealth-consumption process pairs $(Y, C)$ - «distribution» meaning conditionally upon the agent's information, the given filtration $\mathfrak{F}$. We shall assume that consumption choices at time $t$ take effect from time $t^{+}$, such that, ceteris paribus, if $Y^{*}(t)>Y(t)$ the starred process can immediately equalize by consuming the excess; hence the subtracted $\left[C\left(t^{+}\right)-C(t)\right]$ in the following. As we shall only deal with weak orderings, we shall use "preferred to» to include indifference; it is easy to verify that the following indeed forms a partial ordering:

Assumption 3.4: (for the preference orderings) Preferences are weak partial orderings over probability distributions of wealth-consumption pairs, satisfying the following. Given a filtration $\mathfrak{F}$, then for each $\mathfrak{F}$-stopping time $\tau$ the following holds true (interpreting statements on the set $t \geq \tau$ as vacuously true on if $\tau=+\infty)$ :

Consider two wealth-consumption process pairs $\left(Y^{*}, C^{*}\right)$ and $(Y, C)$ on the event that they coincide almost everywhere on $t \in(-\infty, \tau]$, and with $C$ and $C^{*}$ left-continuous. Suppose there exists an $\mathfrak{F}$-predictable a.s. $K$ such that the following equivalence in finitedimensional distributions holds $\mathfrak{F}_{\tau}$-conditionally:

$$
\begin{aligned}
& \left\{\left(Y^{*}(t)-\left[C^{*}\left(t^{+}\right)-C^{*}(t)\right], C^{*}(t)\right)\right\}_{t \geq \tau} \sim \\
& \left\{\left(Y(t)-\left[C\left(t^{+}\right)-C(t)\right], C(t)+K(t)-K(\tau)\right)\right\}_{t \geq \tau}
\end{aligned}
$$


We say that $\left(Y^{*}, C^{*}\right)$ is weakly preferred to $(Y, C)$ (also said to be preferred to $(Y, C)$, interchangeably) at time $\tau$ in either of the following two cases: (i) if $K(t) \equiv K(\tau)$ for all $t>\tau$; (ii) if, in the case where there is not free disposal, cf. Definition 3.6 below, $K$ is nondecreasing. We furthermore say that $\left(Y^{*}, C^{*}\right)$ is preferred to $(Y, C)-i . e$. without any time qualification - if at time 0 it is almost sure that $\left(Y^{*}, C^{*}\right)$ will be preferred to $(Y, C)$ at each finite stopping time $\tau$.

In words, this means that an agent will accept an offer of additional future consumption - i.e. higher $\mathrm{d} C$, not merely higher $C$ ! - for the same future wealth. This is interpreted in probability law, and viewed from any stopping time $\tau$ - the stopping time shall largely be notationally suppressed. Let us give a non-vNM example:

Example 3.5: (A social welfare ordering) Consider as agent a social planner evaluating the welfare of $\theta$ «generations», each with an exponential lifetime of unit intensity, born when the previous dies (i.e., at jump times $\tau_{\theta}$ of a Poisson process), inherits their wealth, and is constrained to keeping wealth nonnegative. Suppose given an increasing function $f$, either lower or upper bounded, such that each generation's welfare is evaluated at time $\tau$ as $w_{\theta}=\mathrm{E}\left[f\left(C\left(\tau_{\theta}\right)-C\left(\tau_{\theta-1}\right)\right) \mid \mathfrak{F}_{\tau}\right]$. Assume the social planner's preferences at time $\tau$ to be leximin over the generations (for an alternative preference ordering: over current and future generations). That is, for each strategy one orders the generations from lowest $w_{\theta}$ to highest, and strategies are then compared lexicographically. See, e.g., Asheim [3] or for issues concerning infinite number of generations: Asheim and Zuber [4]. The lexicograpical element implies that this ordering is not representable as expected utility, so it fails the assumptions of [31]. For the familiar Black-Scholes (geometric Brownian) market treated therein, assumed unrestricted, Theorem 3.12 will show that to each strategy, this social planner will prefer some strategy where one always invests on the Merton line $\left(\Gamma \Gamma^{\top}\right)^{-1} \boldsymbol{\mu}$ - just like the usual expected utility maximizer.

First, however, we will have to make assumptions on the strategies an agent can employ to generate the wealth-consumption process pair. Usually in finance, the strategies are restricted to an admissible subset of possible Itô integrands/integrals, this in order to exclude doubling strategies (cf. Dudley's theorem [13]). We shall not impose one single admissibility concept, rather an assumption on what an admissibility concept can and cannot rule out.

Definition 3.6: (strategies and admissibility) Consider $(\star)$, for a given filtration $\mathfrak{F}$ (satisfying Assumption 3.2). A strategy is a predictable process triplet $(C, D$, v), with $C, D$ leftcontinuous, $D$ nondecreasing, and $\boldsymbol{v}$ (Itô stochastically) locally integrable wrt. $\boldsymbol{\mu} \mathrm{d} t+\Gamma \mathrm{d} \boldsymbol{Z}$ in the sense of Protter [51, Section IV.2, the two definitions following Theorem 14, applied coordinate-wise].

By admissibility we mean the restriction to a given - fixed, but possibly agent-dependent - subset of strategies, called the admissible strategies, such that this subset satisfies all the following:

(a) An admissible strategy must satisfy $v(t) \in \mathbb{V}_{t}$ almost everywhere $(\mathrm{d} t \times \mathrm{dP})$.

(b) Admissibility can require $D \equiv 0$ a.s.; we refer to the case without this $D \equiv 0$ restriction as «free disposal». Admissibility can not impose any other restrictions on $D$, apart from the nondecreasingness required in order to be a strategy.

(c) Let $(C, D, v)$ be any admissible strategy. Then the admissible set must include any $\left(C^{*}, D^{*}, \boldsymbol{v}^{*}\right)$ which coincides with $(C, D, \boldsymbol{v})$ on $(-\infty, 0]$, with $D^{*} \equiv 0$ in the case 
without free disposal, and is such that

$$
\left(\int \boldsymbol{v}^{*}(s)^{\top} \mathrm{d} \boldsymbol{Z}(s), Y^{*}, C^{*}\right) \sim\left(\int \boldsymbol{v}(s)^{\top} \mathrm{d} \boldsymbol{Z}(s), Y, C+K\right)
$$

holds for $K \equiv 0$ if there is free disposal, and for some nondecreasing $K$ vanishing on $(-\infty, 0]$, otherwise.

Admissibility can impose lower (but not upper, in the absence of free disposal) bounds on $\mathrm{d} C$; recall from (1) that $C$ is transformed consumption, and restricting to $\mathrm{d} C \geq-\rho Y \mathrm{~d} t$ imposes nondecreasing actual consumption. Admissibility can also impose arbitrary restrictions on the wealth and the Itô integral part. The reason for including the wealth herein is to facilitate a restriction which appears natural, namely a lower-bound type. This paper will pairwise compare strategies in order to find an improvement to an admissible strategy, and we only need that the improvement strategy be admissible as well.

Note that Definition 3.6 suffices for well-definedness of $Y$ without invoking the theory of stochastic differential equations.

Thus far, the term «agent» has been used loosely. We now restrict the use of the phrase:

Definition 3.7: Fix a particular set of portfolio constraints and an admissibility restriction as in Definition 3.6 (cf. also Assumptions 3.2 (e) and (f)). with $\mathbb{V}$ potentially individual. From now on, the term agent will mean a pair of initial wealth-consumption $\{(Y(t), C(t))\}_{t \leq 0}$ such that an admissible strategy exists and a preference ordering satisfying Assumption 3.4.

Furthermore, we define the same (weak, partial) order on admissible strategies as on the wealth-consumption processes they induce: $\left(C^{*}, D^{*}, \boldsymbol{v}^{*}\right)$ is (weakly) preferred to $(C, D, \boldsymbol{v})$, if the corresponding $\left(Y^{*}, C^{*}\right)$ is (weakly) preferred to $(Y, C)$.

The restriction that admissible strategy exists, may be substantial if for example $\mathbb{V}$ is defined by no shorting and no borrowing; with positive prices, then in order to be an «agent», nonnegative wealth from $0^{+}$is necessary. If in this case admissibility allows to raise money from outside the market immediately, then we can have $Y(0)<0 \leq Y\left(0^{+}\right)$; otherwise, we simply remove these «non-agents» from the market.

We are now ready to define separation, noting that we only compare pairwise, without defining optimality:

Definition 3.8: ( $k$-fund separation) Suppose that there exist $k-1$ predictable $\mathbb{R}^{n}$-valued $\boldsymbol{f}_{1}(t), \ldots, \boldsymbol{f}_{k-1}(t)$ (common to all agents), such that for each admissible strategy $(C, D, \boldsymbol{v})$, there exists an admissible $\left(C^{*}, D^{*}, \boldsymbol{v}^{*}\right)$ which is preferred to $(C, D, \boldsymbol{v})$ by every agent for whom the latter is admissible, and with $\boldsymbol{v}^{*}(t)$ spanned by $\boldsymbol{f}_{1}(t), \ldots, \boldsymbol{f}_{k-1}(t)$, almost everywhere $(\mathrm{d} t \times \mathrm{dP})$. Then we say that the market admits

- $k-1$ fund separation if admissibility implies $\boldsymbol{v}^{*}(t)^{\top} \mathbf{1}=Y(t)$ almost everywhere for every agent, and

- $k$ fund separation - in this case also $k$ fund monetary separation - otherwise.

Remark 3.9: Definition 3.8 does not require linearly independent funds $-k$-fund separation is a special case of $k+1$-fund separation, and $n+1$ fund separation is vacuously true. Furthermore, Definition 3.8 allows for the funds to vary as long as they are common to all agents - even vary stochastically as function of the state of the market, which is what Merton 
[40, Section 15.7] refers to as generalized separation. Merton's generalization is recovered in Section 4.3; for a treatment of the random coefficients case, see also Doukachev [12].

\subsection{The main argument, applied to monetary separation for Brownian noise}

Our main argument will be based upon the approximations defining the Itô integral. As we will only consider Lévy process integrands, the reader can alternatively consult Applebaum [2, sec. 4.3] for an exposition suited for this particular class. The more general construction following and referencing Protter [51] goes in brief as follows: The integral is first defined for bounded predictable integrands (Section II.4) which have sample paths leftcontinuous with right limits and are piecewise constant between times that (Theorem II.21) can be taken nonrandom, e.g., the below choice of dyadic rationals. These can approximate (uniformly on compacts in probability, «ucp») bounded predictables (Theorems IV.2-4ff) and then unbounded $\boldsymbol{v}$ by integrals of $v_{i} 1_{\left\{\left|v_{i}\right| \leq A\right\}}$ (Theorems IV.14ff. - or equivalently $v 1_{\{\|\boldsymbol{v}\| \leq A\}}$ ). A crucial tool is the stochastic dominated convergence theorem (IV.32) which ensures that under stochastic integrability (as in the definition of strategy) the convergence is ucp; thus, equivalence in law is preserved when we take the limits of approximate strategies as defined in the following. Notice that the «null» portfolios in the approximation is merely a technicality in order to control the events when $\mathbb{V}$ has large distance to the origin.

Definition 3.10: By a partition $\varpi$ of the time half-line, we mean an $N \in \mathbb{N}$ and the dyadic rationals $T_{d}=d \cdot 2^{-N}$ for $d=0, \ldots, 4^{N}$. We say that partitions refine to mean that $N \rightarrow+\infty$. For a given admissible strategy $(C, D, v)$, the term approximating strategies means a sequence of refining partitions $\varpi$, each with a step strategy $\left(C_{\varpi}, D_{\varpi}, \boldsymbol{v}_{\varpi}\right)$ with $\boldsymbol{v}$ constant $\left(T_{d}, T_{d+1}\right]$ with and either $=\mathbf{0}$ or $\in \mathbb{V}_{T_{d}^{+}}$and with Euclidean norm $\leq N$; furthermore, the sequence of corresponding wealth-consumption processes $\left(Y_{\varpi}, C_{\varpi}\right)$ converges to $(Y, C)$ ucp as partitions refine.

This ucp convergence implies convergence in law. Once we have established existence of approximating strategies, it suffices to show separation for these.

Lemma 3.11: Every admissible strategy admits approximating strategies.

Furthermore, fix a $k$ and suppose for each $t$ that $\mathbb{V}_{t}$ is a closed intersection between a radial set and a hyperplane. Suppose in addition that for each partition $\varpi$ that there exists $k$ risky funds, constant between the dyadic rationals in the partition, and satisfying the following: For each partition $\varpi$ and each step strategy $\left(C_{\varpi}, D_{\varpi}, \boldsymbol{v}_{\varpi}\right)$ - with all $\boldsymbol{v}\left(T_{d}^{+}\right) \in \mathbb{V}_{T_{d}} \cup\{\mathbf{0}\}$ and bounded - there exists a step strategy $\left(C^{*}, D^{*}, v^{*}\right)$, where all $\boldsymbol{v}^{*}\left(T_{d}^{+}\right)$are bounded, $\in \mathbb{V}_{T_{d}} \cup\{\mathbf{0}\}$ and spanned by the $k$ risky funds, such that the for the corresponding wealthconsumption pairs, $\left(Y_{\varpi}^{*}, C_{\varpi}^{*}\right)$ is preferred to $\left(Y_{\varpi}, C_{\varpi}\right)$.

Then the market admits $k+1$ fund monetary separation if a safe opportunity exists (and $k$ fund separation if not).

Apart from a few technicalities, the proof consists in comparing distributions for the approximations, and taking note that convergence in probability implies convergence in distribution.

Thus, by assing to approximating strategies, we can see that a static argument suffices: we can improve pairwise for each time without intertemporal trade-off. The following separation theorem is essentially the case of [31] with our more general preference assumptions. 
Assuming $\boldsymbol{Z}$ to be a Wiener process without drift, we can and will without loss of generality assume it to be standard Brownian, by redefining $\Gamma$.

Theorem 3.12: (Two-fund monetary separation, Black-Scholes market) Suppose constant market coefficients, and that $\boldsymbol{Z}$ is standard Brownian. Assume the set $\mathbb{V}_{t}$ to be piecewise constant, nonrandom, and for each $t$ is a closed radial set such that $\boldsymbol{x}^{\top} \Gamma \Gamma^{\top} \boldsymbol{x}>0 \forall \boldsymbol{x} \in$ $\mathbb{V}_{t} \backslash\{\mathbf{0}\}$. Then the market admits two-fund monetary separation, using for each $t$ a scaling of $\boldsymbol{f}(t)=\operatorname{argmax}\left\{\boldsymbol{x}^{\top} \boldsymbol{\mu} ; \boldsymbol{x} \in \mathbb{V}_{t}\right.$ and $\left.\boldsymbol{x}^{\top} \Gamma \Gamma^{\top} \boldsymbol{x}=1\right\}$ (if the argmax is non-unique, chosen so that $t \mapsto f$ is Borel).

The proof itself is given in Appendix 1, but let us explain it. We shall «re-specify» strategies in «feedback» form and back to $(t, \omega)$-dependence, and we consider feedback form only for the approximations, where we do not need to invoke any differential equation theory. We want to improve the portfolio and increase consumption, but not statewise: it needs to relate to the wealth process the new strategy generates. The way to do so, is to (i) pass to an approximating strategy, (ii) re-specify that in feedback form, so that we get a stochastic difference equation where existence and uniqueness are trivial, (iii) improve in feedback form, and (iv) write as function of $(t, \omega)$ before we (v) refine the partitions to the limit. Note that the «feedback» in step (ii) might need to invoke randomization. The filtration includes enough independent information that the agent could randomize - this by assumption, but unavoidable anyway with $Z$ being Brownian: there will for each $\boldsymbol{x} \in \mathbb{R}^{n}$ be an $n-1$-dimensional Wiener process orthogonal to and thus independent of $\boldsymbol{x}^{\top} \Gamma \mathrm{d} \boldsymbol{Z}$. We resolve this in (ii) ff. by applying Assumption 3.2(c), to let the choice also depend on an $\mathfrak{H}_{T_{d}}^{(Y, C, M)} \vee \mathfrak{S}^{\boldsymbol{L}\left(T_{d}\right)}$-measurable random variable of $\mathfrak{H}_{T_{d}}^{(Y, C, M)}$-conditionally same distribution. The reader should interpret «feedback» accordingly (and in the proof of Theorem 4.5, involve the state of $M$ ).

The essence is the following recipe: define $Q_{\boldsymbol{x}}=\boldsymbol{x}^{\top} \Gamma \Gamma^{\top} \boldsymbol{x}$ and improve any $\boldsymbol{v}$ which does not solve the static problem

$$
\max _{\boldsymbol{x} \in \mathbb{V}_{t}} \boldsymbol{x}^{\top} \boldsymbol{\mu}(t) \quad \text { s.t. } \quad Q_{\boldsymbol{x}}=Q_{\boldsymbol{v}(t)}(t)
$$

by replacing it with one which does; this is the mutual fund $Q_{\boldsymbol{v}(t)} \boldsymbol{f}$, where $\boldsymbol{f}=\boldsymbol{f}(t)$ if $\mathbb{V}$ or the market coefficients depend on time.

Remark 3.13: This recipe assumes, through positive definiteness on $\mathbb{V}$, absence of «static» arbitrage opportunities, but does not eliminate doubling strategies. We facilitate an admissibility concept that can rule them out, but not for the purpose of the technique: even such strategies can be compared pairwise for improvement.

\section{Separation with elliptical distributions}

This section will give separation theorems when the driving noise is an elliptical Lévy process (in particular applying to Brownian motion), with or without a risk-free opportunity. An elliptical distribution is characterized nicely by «mean» and «covariance» (suitably interpreted), and a univariate radial variable. The ellipticals constitute the class of distributions to do proper mean-variance trade-off on (cf. Owen and Rabinovich [49] and Chamberlain [10]; see also e.g. the book of Gupta et al. [25]). We will first review the essentials of the distribution class, and relate it to Lévy processes. 


\subsection{Elliptical distributions and spherical Lévy processes}

Recall that a random vector $\boldsymbol{X}$ is elliptical, (also known as elliptically contoured), if its characteristic function can be represented as

$$
\mathrm{E}\left[\exp \left(\mathrm{i} \boldsymbol{\xi}^{\top} \boldsymbol{X}\right)\right]=\exp \left(\mathrm{i} \boldsymbol{\xi}^{\top} \boldsymbol{\mu}\right) h\left(\sqrt{\boldsymbol{\xi}^{\top} \Gamma \Gamma^{\top} \boldsymbol{\xi}}\right)
$$

for some function $h$ (necessarily real), some location vector $\mu$ and some matrix $\Gamma$ - see e.g. Cambanis et al. [8]. It intentional to use the same symbols as the market coefficients of $(\star)$; in the next subsection, we shall use this distribution for $\boldsymbol{Z}(1)$. Such a random variable $\boldsymbol{X}$ admits the representation

$$
X \sim \mu+\Gamma S \sqrt{R}
$$

where the random vector $S$ is uniform on the unit sphere in the appropriate-dimensional Euclidean space, and the radial scaling variable $\sqrt{R}$ is independent of $\boldsymbol{S}$. Within the theory of elliptical distributions, it is common to refer to $\mu$ and $\Gamma \Gamma^{\top}$ as «mean» and "covariance» regardless of integrability; we have $\mathrm{E}[X]=\mu$ iff $\mathrm{E} \sqrt{R}<\infty$, and we have $\mathrm{E}\left[(\boldsymbol{X}-\boldsymbol{\mu})(\boldsymbol{X}-\boldsymbol{\mu})^{\top}\right]=\Gamma \Gamma^{\top} \mathrm{E} R / n$. The key to the separation property is the distribution of linear combinations of the coordinates (a.k.a. «projections»): by rotational invariance, the distribution of $\boldsymbol{x}^{\top} \Gamma \boldsymbol{S}$ depends only on the Euclidean norm of $\boldsymbol{x}^{\top} \Gamma$. Thus - for fixed distribution of $R$ - the distribution of $\boldsymbol{x}^{\top} \boldsymbol{X}$ depends only on $\boldsymbol{x}^{\top} \Gamma \Gamma^{\top} \boldsymbol{x}$ and $\boldsymbol{x}^{\top} \boldsymbol{\mu}$.

The $S \sqrt{R}$ part is called spherical, and the probability measure of a spherical variable is sometimes called isotropic: the probability of a given set, is invariant under rotations about the origin. Both the characteristic function and the measure are then norm-dependent, which is in fact a unique feature to the weighted Euclidean norms. Letting the market coefficients take care of the affine transformation of (8), we shall focus on the Lévy measure - the intensity of jumps of amplitude $\boldsymbol{\delta}$ - of a spherically distributed Lévy process $\boldsymbol{Z}$. There seems not to be much mention in the literature on isotropic Lévy measure, so we give the following easy result, with a proof in Appendix 1 :

Lemma 4.1: Assume that that $\boldsymbol{Z}$ - as per Assumption 3.2 - is a Lévy process with zero drift. Suppose that its Lévy measure $\lambda$ is isotropic and its Gaussian part is a scaling $\sigma$ of standard Brownian motion. Then the increments of $\boldsymbol{Z}$ are spherically distributed.

Remark 4.2: Should $\Gamma$ be the identity, then the self-financing model without arbitrage or redundant opportunities, has the following simple necessary and sufficient condition that prices a.s. stay positive, thus being useful to model limited liability assets: $\lambda$ vanishes outside the closed unit ball. Notice that since $\lambda$ is isotropic, the jumps of amplitude 1 will almost surely not be in a direction that would send a given price to zero. If there on the other hand is mass outside the unit ball, then each of the prices will a.s. switch sign sooner or later. With general $\Gamma$, some prices could change sign while others do not; the question reduces to whether and where the ellipsoid centered at $\boldsymbol{\mu}$, intersects the complement of the closed positive orthant. 


\subsection{Separation properties}

In this section, we shall assume that the increments of $\boldsymbol{Z}$ are spherical, so that $\Gamma \mathrm{d} \boldsymbol{Z}(1)$ has covariance matrix $\Xi$ being a positive scaling of $\Gamma \Gamma^{\top}$. The distribution of the increment $\boldsymbol{v}(t)^{\top} \Gamma(t) \mathrm{d} \boldsymbol{Z}$ is then completely characterized by instantaneous drift $\boldsymbol{v}(t)^{\top} \boldsymbol{\mu}(t)$ and instantaneous variance $\boldsymbol{v}(t)^{\top} \boldsymbol{\Xi}(t) \boldsymbol{v}(t)$. We leave to the reader to verify that the proofs apply to the «relaxed» version of Assumption 3.2(d) where coefficients are time-dependent deterministic, requiring, of course, that the assumptions of the theorems hold for each $t-$ this generality will be notationally suppressed.

For the construction analogous to the Gaussian unconstrained case of Section 3.1, it therefore suffices that the portfolio $\boldsymbol{v}^{*}(t)$ maximizes $\boldsymbol{x}^{\top} \boldsymbol{\mu}$ given that $\boldsymbol{x}^{\top} \boldsymbol{\Xi} \boldsymbol{x}$ equals $\boldsymbol{v}(t)^{\top} \boldsymbol{E} \boldsymbol{v}(t)$ - the existence of maximizer is granted if there is no arbitrage, i.e. if no investment opportunity can be written in terms of the others plus excess drift. A precise condition for absence of arbitrage is not equally straightforward when e.g. shorting is forbidden on one or more of the opportunities, which is a special case of part (a) of the following theorem; we assume for sake of simplicity positive definite $\Xi$.

Theorem 4.3: (Two-fund separation and extensions) Consider the dynamic model ( $\star$ ) with $\boldsymbol{Z}$ having spherical increments. Suppose $\Gamma \Gamma^{\top}$ positive definite, and assume precisely one of the following three conditions:

(a) $\mathbb{V}$ is closed and radial and common to all agents, or

(b) $\mathbb{V}_{t}=\left\{\boldsymbol{v} ; \boldsymbol{v}^{\top} \mathbf{1}=Y(t)\right\}$ i.e. no risk-free opportunity, or

(c) $\mathbb{V}$ is the intersection of a convex cone common to all agents (and with vertex at $\mathbf{0}$ ), and the agent-specific affine hyperplane in (b), and $\boldsymbol{\mu}=\bar{\mu}_{0} \mathbf{1}=\mathbf{0}$ cf. Assumption 3.2(f).

Then the market admits two-fund separation (monetary in case (a)).

(d) Part (b) is a special case of this part: Suppose that $\mathbb{V}_{t}$ is defined by the linear constraints

$$
\boldsymbol{v}(t)^{\top} \zeta_{j} \leq z_{j} \quad j=1, \ldots, \bar{k}
$$

where the $\zeta_{j}$ are common to all agents, but the $z_{j}$ allowed to be individual. Let $k+1$ be the number of linearly independent vectors in the set $\left\{\boldsymbol{\mu}, \zeta_{1}, \cdots, \zeta_{\bar{k}}\right\}$. Then we have $k+1$-fund separation if (9) forbids risk-free investment, and $k+2$-fund monetary separation otherwise.

(e) Consider the assumptions of part (d). Modify the model by imposing a leveragedependent and possibly agent-dependent running cost rate («credit spread») $\hat{\rho}(b(t)) \mathrm{d} t$ associated to the agent's choice of $b(t):=v(t)^{\top} \mathbf{1}$.

We then have $k+3$-fund separation (in particular, 3-fund separation if there are no constraints in (9)).

The proof goes like Theorem 3.12 for part (a); the other parts follow likewise, though with a Kuhn-Tucker program with with one quadratic constraint and linear ones. See Appendix 1.

Remark 4.4: In related literature, like Ross [53, Section 1], the fund $\boldsymbol{f}_{2}$ in the cases (b) and (c) without risk-free opportunity, is absent. The reason is risk aversion, which we do not assume. Hence we do not have «one fund separation»: we need $\boldsymbol{f}_{2}$ to inflate volatility. 


\subsection{Generalized separation; market parameters driven by $Z$ through $M$}

Thus far we have relaxed Assumption 3.2(d) no further than to deterministic time-dependence. Khanna and Kulldorff [31] point out that coefficients may be stochastic, as long they are as stochastically independent of $\boldsymbol{Z}$. The reason why dependence is prone to destroy separation properties, is that the agent will have to consider the joint distribution of the (tomorrow's wealth, tomorrow's market) pair. Indeed, the dynamics of the underlying investment opportunities assumed so far - geometric for the traded market justifiction ensures that neither wealth nor state of the market, depends on any of the prices levels $P_{i}$. If one parameter were to depend on the cardinal level of prices, then we would have to enter this as a state variable - this is the purpose of the market state $M$.

Merton [40, Section 15.7] allows for a univariate market state variable and deduces the need for another fund. His choice of using the interest rate is done for interpretability, and admits generalizations as long as it is driven by $\boldsymbol{Z}$ - possibly accommodated by augmenting $\boldsymbol{Z}$ to a higher dimension. Assume in this subsection $(Y, M)$ to obey the dynamics

$$
\mathrm{d}\left(\begin{array}{c}
Y(t) \\
M(t)
\end{array}\right)=\left(\boldsymbol{v}^{\top}, 1\right)\left[\left(\begin{array}{c}
\boldsymbol{\mu}(t, M(t)) \\
\bar{\mu}(t, M(t))
\end{array}\right) \mathrm{d} t+\left(\begin{array}{c}
\Gamma\left(t, M\left(t^{-}\right)\right) \\
\boldsymbol{\gamma}^{\top}\left(t, M\left(t^{-}\right)\right)
\end{array}\right) \mathrm{d} \boldsymbol{Z}\right]-\left(\begin{array}{c}
\mathrm{d} C(t) \\
0
\end{array}\right)
$$

For given time and state, the instantaneous covariance matrix of $\mathrm{d}(Y, M)^{\top}$ depends on $\boldsymbol{x}$ not only through $\boldsymbol{x}^{\top} \Gamma \Gamma^{\top} \boldsymbol{x}$, but also through $\boldsymbol{x}^{\top} \Gamma \boldsymbol{\gamma}$. In constructing a dominating strategy will have to take into account that the agent's preferences involves the law of the future wealth-consumption-opportunity set triplet, and by the strong Markov property, the opportunity set is representable through the history-less state of $M(t)$. The reader can note that the generalization to higher-dimensional $M$ is straightforward, at the cost of requiring more funds:

Theorem 4.5: (Generalized separation) Modify the setup of Theorem 4.3 by generalizing the dynamics ( $\star$ ) into (10), and that there exists a unique M satisfying (10).

Then Theorem 4.3 part (d) applies as if (9) were augmented with the additional constraint $\boldsymbol{x}^{\top} \Gamma \boldsymbol{\gamma}=\bar{z}$, and with funds depending on $(t, M(t))$ as well.

The proof works by fixing the value of $\boldsymbol{x}^{\top} \Gamma \boldsymbol{\gamma}$ to $\bar{z}=\boldsymbol{v}(t)^{\top} \Gamma \boldsymbol{\gamma}$ for the strategy to be improved; that is a linear constraint, and we adapt the relevant part of Theorem 4.3. See Appendix 1. Notice that if $\Gamma \boldsymbol{\gamma}=\mathbf{0}$, then $\mathrm{d} M$ is instantaneously uncorrelated with portfolio return, and the additional fund vanishes; this holds also for non-Gaussian ellipticals, where uncorrelated does not imply independent. To sum up, the following generalizations behave quite uncomplicated under ellipticity: Introducing a(nother) linear constraint $\sim$ another fund. Introducing a common (correlated) risk factor $\leadsto$ another fund. It is also shown in [19] - in a single period model - that the partial information that one of the univariate projections of $\boldsymbol{Z}$ is $\geq$ a certain level, also leads to one more fund being required. Actually, full information removes it, provided the market is still free of arbitrage - we can then rebuild the model with one less risk factor.

\section{5. «No short sale» admits skewed separating distributions: the $\alpha$-stable laws}

There is a conceptually simple extension of the definition (7) of ellipticity, namely to allow $h$ to take as input a more general (quasi-) norm of $\xi$. This leads to the so-called 
pseudo-isotropic distributions, see [20] for single period portfolio theory; this class contains the symmetric $\alpha$-stable laws. There are however non-symmetric stable distributions for which we can adapt Theorem 4.3 under no short sale. The stable distributions form an infinitely divisible class generalizing the Gaussian, and have been applied in widely in financial modelling in cases where the Gaussian exhibits too light tail, see, e.g., [32, chapters E1, E14 and E16]), [36], [43], [52] and [28]. The treatment of stable distributions in the finance literature is often confined to a restricted subclass of the symmetric ones. For example, the classical works of Samuelson [56], Fama [14] and Ross [53] assume a dependence structure described by matrix multiplication of iid's. Alternatively, as multivariate versions of univariate laws can be generated by ellipticity through choosing the radial scaling, several authors focus on the elliptical stable laws, e.g., in a setting not unlike this paper, Ortobelli et al. [46,47]. More general treatments of symmetric stable distributions appear ([21], [5], [7]), but the skewed stable laws are commonly disregarded in the portfolio theory literature. Obviously, skewed distributions do not admit the construction of the previous section when the portfolio can be chosen over all of $\mathbb{R}^{n}$. However, it may apply when restricting to e.g. the first orthant, which we shall exploit; these are results that the author has struggled to find in the literature, even in the single period model, apart from the most basic observations.

Essentials on stable distributions can be found in Applebaum [2, Section 1.2.5] and - as of writing - for the univariate case in Nolan's online chapter [44]; for a reference work, see Samorodnitsky and Taqqu [55]. The common defining property of a stable random vector $\boldsymbol{X}$ in $\mathbb{R}^{n}$, is that for two independent copies $\boldsymbol{X}_{1}$ and $\boldsymbol{X}_{2}$ of $\boldsymbol{X}$, and any two positive numbers $a_{1}$ and $a_{2}$, there exist non-random $a \geq 0$ and $\boldsymbol{d} \in \mathbb{R}^{n}$ such that $a_{1} \boldsymbol{X}_{1}+a_{2} \boldsymbol{X}_{2} \sim a \boldsymbol{X}+\boldsymbol{d}$. As it turns out, $a^{\alpha}=a_{1}^{\alpha}+a_{2}^{\alpha}$ for some $\alpha \in(0,2]$, hence the term « $\alpha$-stable», with $\alpha=2$ being the already treated Gaussian. We shall however have more use for the characteristic function. Of many representations (see Nolan [44] or Hall [26]) we choose the following: there exists a (unique if $\alpha<2$ ) finite spectral measure $\varkappa$ on the unit sphere $\mathbb{S}$ such that, with $w(x, \alpha)=\tan (-\alpha \pi / 2)$ for $\alpha \neq 1$ and $w(x, 1)=\frac{2}{\pi} \ln x$, we have

$$
\mathrm{E}\left[\exp \left(\mathrm{i} \boldsymbol{\xi}^{\top} \boldsymbol{X}\right)\right]=\exp \left(\mathrm{i} \boldsymbol{\xi}^{\top} \boldsymbol{\mu}-\int_{\mathbb{S}}\left|\boldsymbol{\xi}^{\top} \boldsymbol{s}\right|^{\alpha}\left[1+\mathrm{i} w\left(\left|\boldsymbol{\xi}^{\top} \boldsymbol{s}\right|, \alpha\right) \operatorname{sign}\left(\boldsymbol{\xi}^{\top} \boldsymbol{s}\right)\right] \varkappa(\mathrm{d} \boldsymbol{s})\right)
$$

For $\alpha<2, \boldsymbol{X}-\boldsymbol{\mu}$ is symmetric iff $\varkappa$ is symmetric. Unlike the characterization (7) of ellipticals, there is no « $\Gamma$ » matrix involved in (11); the dependence structure captured by $\varkappa$ is richer, and can be represented by a matrix only iff $\varkappa$ is supported by a finite number of points. We therefore assume - without loss of generality - that

$$
\Gamma=\text { the identity. }
$$

With $\boldsymbol{X}$ as in (11), each linear combination $\boldsymbol{x}^{\top} \boldsymbol{X}$ is univariate stable, with distribution fully determined by the following triplet - note the $1_{\{\alpha=1\}}$ in the location - where from now on we notationally suppress that we integrate over the entire support of the measure:

$$
\begin{aligned}
\text { scale: } \sigma_{\boldsymbol{x}}= & \left(\int\left|\boldsymbol{x}^{\top} \boldsymbol{s}\right|^{\alpha} \varkappa(\mathrm{d} \boldsymbol{s})\right)^{1 / \alpha}, \quad \text { skewness: } \beta_{\boldsymbol{x}}=\sigma_{\boldsymbol{x}}^{-\alpha} \int\left|\boldsymbol{x}^{\top} \boldsymbol{s}\right|^{\alpha} \operatorname{sign}\left(\boldsymbol{x}^{\top} \boldsymbol{s}\right) \varkappa(\mathrm{d} \boldsymbol{s}) \\
& \text { and location: } \mu_{\boldsymbol{x}}=\boldsymbol{x}^{\top} \boldsymbol{\mu}-1_{\{\alpha=1\}} \cdot \frac{2}{\pi} \int \boldsymbol{x}^{\top} \boldsymbol{s} \cdot \ln \left|\boldsymbol{x}^{\top} \boldsymbol{s}\right| \varkappa(\mathrm{d} \boldsymbol{s}) .
\end{aligned}
$$


The Lévy measure is on the form $c_{ \pm} \cdot|z|^{-1-\alpha} \mathrm{d} z$ for $\pm z>0$. Both tails are heavy, except for the totally skewed univariate distributions with skewness $= \pm 1$ and $c_{+} c_{-}=0$ in the Lévy measure: those have either only upwards or only downwards jumps. Indeed, for $p>0$ and $\alpha<2$ we have $\mathrm{E}\left[\min \left\{0, \boldsymbol{x}^{\top} \boldsymbol{X}\right\}^{p}\right]<\infty$ only iff $p<\alpha$ or $\beta_{\boldsymbol{x}}=-1-$ in which case, the moment-generating function converges as well. This case is potentially interesting to applications, to be discussed in Section 6.1.

Thus compared to the ellipticals, the stable laws yield a third parameter to consider, and in Counterexample 5.4 we shall see that it is impossible to accommodate this with a third fund - this partially answering a conjecture found at McCulloch's website [37]. This issue degenerates if $\beta_{\boldsymbol{x}}$ happens to be constant over the opportunity set: one such case is if the investment opportunities are independent with the same skewness and short sale is forbidden. This is known, see again [37], though it rarely appears in the literature. Nonindependent marginals will easily void the crucial properties; e.g., using a $\varkappa$ supported by merely three points, one easily constructs a bivariate $\boldsymbol{X}$ with symmetric marginals such that $\beta_{\boldsymbol{x}}$ varies over the positive quadrant. The following theorem covers both shifted symmetric distributions (in wider generality, part (a)) and special non-symmetric cases where skewness is in fact invariant over an orthant (part (b)). For the latter, formula (14) must be well known to specialists on stable distributions, but the author has not seen the condition applied to portfolio theory.

Theorem 5.1: (Two-fund monetary separation)

(a) Suppose in this part that $\boldsymbol{X}-\boldsymbol{\mu}$ is pseudo-isotropic, i.e. we have the following generalization of symmetric $\alpha$-stability and of ellipticity: $\mathrm{E}\left[\exp \left(\mathrm{i} \xi^{\top}(\boldsymbol{X}-\boldsymbol{\mu})\right)\right]=$ $g(\varsigma(\xi))$, where $g$ and $\varsigma$ are real functions, $\varsigma$ positive-homogeneous of degree 1 , and $\boldsymbol{\varsigma}>0$ except $\varsigma(\mathbf{0})=0$. Let $\mathbb{V}$ be any closed radial, and let $\boldsymbol{f}$ maximize $\boldsymbol{x}^{\top} \boldsymbol{\mu}$ subject to $\{\boldsymbol{x} \in \mathbb{V} ; \boldsymbol{\varsigma}(\boldsymbol{x})=1\}$. Then for each $\boldsymbol{x} \in \mathbb{V}$, the random variable $\varsigma(\boldsymbol{x}) \boldsymbol{f}^{\top} \boldsymbol{X}$ first-order stochastically dominates $\boldsymbol{x}^{\top} \boldsymbol{X}$.

(b) Let $\boldsymbol{X}$ be $\alpha$-stable, satisfying (11) (and thus (13)). Let $\mathbb{H}$ be any closed orthant, possibly rotated but with vertex at the origin, and denote by $-\mathbb{G}$ the set $\{\boldsymbol{x} ;-\boldsymbol{x} \in \mathbb{G}\}$.

Then for each $\beta \in[-1,1]$, the set $\left\{\boldsymbol{x} \in \mathbb{R}^{n} ; \beta_{\boldsymbol{x}}=\beta\right.$ or $\left.\boldsymbol{x}=\mathbf{0}\right\}$ is closed and radial, and it contains $\mathbb{H}$ if $\varkappa$ can be written as a convex combination

$$
\varkappa(\mathbb{G})=\frac{1+\beta}{2} \bar{\varkappa}(\mathbb{G})+\frac{1-\beta}{2} \bar{\varkappa}(-\mathbb{G})
$$

for some finite measure $\bar{\varkappa}$ which vanishes outside $\mathbb{H} \cap \mathbb{S}$.

Furthermore, if $\mathbb{V}$ is any closed radial on which $\beta_{\boldsymbol{x}} \equiv \beta$, and $\boldsymbol{f}$ maximizes the location $\mu_{\boldsymbol{x}}$ of (13) subject to $\left\{\boldsymbol{x} \in \mathbb{V} ; \sigma_{\boldsymbol{x}}=1\right\}$, then for each $\boldsymbol{x} \in \mathbb{V}$, the random variable $\sigma_{\boldsymbol{x}} \boldsymbol{f}^{\top} \boldsymbol{X}$ first-order stochastically dominates $\boldsymbol{x}^{\top} \boldsymbol{X}$.

(c) Modify the assumptions of Theorem 4.3 part (a) such that $\boldsymbol{\mu}+\boldsymbol{Z}(1) \sim$ the $\boldsymbol{X}$ of either part (a) or part (b) of this theorem, with $\mathbb{V}$ as therein. Then the continuous-time market admits two-fund monetary separation.

Part (a) is [20, Theorem 9], and it is a direct generalization of Theorem 4.3 part (a). It also generalizes the subcase of Theorem 5.1 part (b) where $\beta=0$ in (14), and it is proven just like those two cases. The rest of the theorem will also follow analogous to the elliptical case, except some additional calculations in the asymmetric oddball case $\alpha=1$. That 
case admits a peculiar one-fund separation result, to the best of the author's knowledge unpublished even for the single period model:

Corollary 5.2: Assume $\mathbb{H}=\mathbb{R}_{+}^{n}, \alpha=1$ and that $\varkappa$ satisfies (14) with $\int s \mathrm{~d} \bar{\varkappa}=\bar{\zeta} \mathbf{1}$ for some $\bar{\zeta}>0$ (if $\beta \neq 0$, this is equivalent to $\int s \mathrm{~d} \varkappa=\zeta_{0} \mathbf{1}$ for some $\zeta_{0} \neq 0$ ). Modify the assumptions of Theorem 5.1 part (b) by restricting $\mathbb{V}$ further by removing the risk-free opportunity.

Then an admissible strategy must satisfy $\sigma_{v}=\bar{\zeta} Y$, and the static argument from Theorem 5.1 part (b) yields the unique choice $\bar{\zeta} Y \boldsymbol{f}$, with the same $\boldsymbol{f}$ as therein.

One-fund separation carries over to the analogous modification (i.e. removing the riskfree opportunity) of Theorem 5.1 part (c), but if admissibility requires wealth to remain a.s. nonnegative for all $t$, positive-wealth agents must consume everything at once except iff $\beta=1$. If on the other hand it is admissible to raise money through negative consumption $\mathrm{d} C(\{t\})=Y(t)$, then all agents have an admissible strategy.

So in this case, only $\beta=+1$ leaves an agent at positive value for all times. The admissibility of cashing out negative wealth by negative consumption, should be interpreted as the ability to raise money from outside the market; if $\mathrm{d} C=0$ is always admissible, it suffices that the agent has this opportunity once (only to stay at $Y=0$ from then on).

Remark 5.3: A few remarks:

(a) Like for ellipticals, the results apply verbatim if we assume $Z(1) \sim(X-\mu) \sqrt{R}$ in place of $(\boldsymbol{X}-\boldsymbol{\mu})$ - implicitly assuming infinite divisibility (which is not a trivial matter, see e.g., Misiewicz [41,42]).

(b) What if there is no risk-free opportunity? Fama [15, Section VI.B] comments that the assumption of risk-free opportunity "greatly simplifies determination of the efficient set of portfolios». Indeed, we no longer have two-fund separation - not even three fund separation under symmetry: [20, Theorem 11] gives counterexamples and weaker results available for narrow sub-class of the pseudo-isotropic distributions, including iid stables for a discrete set of $\alpha$ 's. (Also certain non-integrable cases admit corner solutions.)

(c) We shall not dwell at the cases with $\alpha<1$ except warning that the totally skewed distributions are then supported by a half-line, and one must be careful about the implications of the no-arbitrage assumption.

We end the discussion on stable distributions with a negative result promised earlier in the paper: not for any $\alpha \in(1,2)$ is there a general three-fund separation theorem for non-symmetric distributions, even if we assume that a risk-free opportunity exists.

Counterexample 5.4: (No three-fund monetary separation with skewness free of choice) Fix an arbitrary $\alpha \in(1,2)$. Assume a risk-free opportunity exists, and in addition there are three independent risky opportunities, $\alpha$-stable with scale normed to unity and expectations $\mu_{i}>0$. First-order stochastic dominance does not order a pair with different scale, nor a pair with different skewness. Hence we need the risk-free opportunity as a fund, and if opportunity \#1 is the only with skewness $\in\{-1,1\}$, we need this as a fund too. Assume now that the respective skewnesses are $1,1 / 2$ and 0 , and that the $\mu_{i}$ satisfy $\mu_{1}=\eta \cdot 2^{(2-\alpha) / \alpha} \mu_{2}$ and $\mu_{3}=(1-\eta) \cdot 2^{(2-\alpha) / \alpha} \mu_{2}$, some $\eta \in(0,1)$. Consider three agents who want skewnesses $1,1 / 2$ and 0 for their respective portfolios, and suppose the latter wants unit scale. The former agent requires the fund $(1,0,0)^{\top}$. The second will 
choose a scaling of $(1,1,1)^{\top}$, from the construction of the $\mu_{i}$. For three-fund separation, the last agent must choose $v_{2}=v_{3}$; zero skewness implies $v_{1}=-2^{-1 / \alpha} v_{2}$, and the agent thus maximizes $(1-\eta) x_{3}+\left(1-2^{-1 / \alpha} \eta\right) x_{2}$ subject to $\frac{3}{2}\left|x_{2}\right|^{\alpha}+\left|x_{3}\right|^{\alpha}=\sigma$. Only iff $\eta=\left(3 \cdot 2^{1 / \alpha}-2\right)^{-1}$ the solution has $x_{2}=x_{3}$ and is in the span of the funds; otherwise, we need all opportunities.

\section{Discussion}

Let us first, briefly, mention a couple of cases left out. First, we have in the interest of length omitted special results for stable distributions with $\alpha<1$. Second, as the interesting distribution is that of the portfolio returns, not of the individual returns, one could ask whether there are non-stable vectors with all projections stable. Such exist (found by Marcus, [33]), but unsuitable for the Lévy process model, as they cannot be infinitely divisible - nor integrable. See [24, Theorem 2.3] and [55, Section 2.2, Theorems 2.1.5 and 3.1.2].

\subsection{Possible consequences for applications}

The extension of two-fund monetary separation to $\alpha$-stability, can be regarded as a robustness result; if one is reasonably close to applying the central limit theorem, and the distributions are symmetric, we could expect the mutual fund property to be a reasonable model. We have seen that the property is robust to skewness as long as that is invariant over the opportunity set, and given conditions for this also with dependent marginals (formula (14)). It is not unreasonable in insurance that profits/losses have a light upper tail and a heavy downside tail, accommodated by putting $\beta=-1$ in (14) and attempting to adapt Theorem 5.1 parts (b) ff. Even if insurance policies are not shared between the companies, a mutual fund-type theorem may apply to the distributions they choose, leading to insurers targeting the same loss distributions up to scaling, and thus equivalent (in joint distribution) customer mass. The same reasoning applies to a loan portfolio, which is also an application where it is reasonable to assume asymmetry and a lighter upper than lower tail.

What about an insurance company's asset/liability management? What about a financial conglomerate exposed also to market risk, trading in assets which have plausibly symmetric returns, and plausibly with different tail heaviness/index of stability? Say, there is an insurance portfolio with asymmetric profit/loss as above, and the firm invests its assets in part in a stock market with symmetric returns, and holding the rest risk-free? From Counterexample 5.4 we have no hope for a two-fund theorem even if these markets have the same $\alpha$. If we have risk-free opportunity and each market satisfies the conditions of Theorem 5.1 (which then subsumes the ellipticals), we can do with the safe opportunity and one risky fund per market. With stochastic dependencies, on the other hand, we are in difficulties as soon as we leave the elliptical class - or even if the «blocks» are each elliptical. It certainly does not become simpler if we assume dependence between e.g. a stock market being a geometric Brownian Black-Scholes market, and non-elliptical insurance portfolios. 


\subsection{Concluding remarks}

This paper has shown how Ross-type portfolio separation in continuous-time models is easily inherited from static models without dynamic programming. In this sense, the single period model is more general than the continuous-time model, since we assume the restriction to infinite divisibility. The transition from static to dynamic model to dynamic model can be done with little if any stochastic calculus - not even the second-order term from the Itô formula needs to be introduced. Rather, one utilizes basic tools of probability theory and, from analysis, the standard way of defining an integral by simple functions and extension by analytic continuation. The modest requirements are due the separation theorem trying only to reduce the dimensionality of the portfolio choice problem, rather than finding, or even defining, an optimal strategy.

In this paper, we have used the method to easily recover the separation property for classical cases, and extend these under no-shorting portfolio restrictions, facilitating certain non-symmetric returns distributions. While this may be of interest for modelling other financial activities than the trading portfolio perspective, there are also challenges due to the rich dependence structures possible, and thus room for future research on generalizations of this celebrated theorem.

\section{Acknowledgements}

The work was initiated at the Stockholm School of Economics, a stay supported by NorFA; thanks to Tomas Björk for introducing me to the idea and the Khanna/Kulldorff approach. During the research, the author has been affiliated with The Financial Supervisory Authority of Norway; the usual disclaimer applies. The paper has benefited from referee comments. All errors are mine.

\section{Disclosure statement}

No potential conflict of interest was reported by the author.

\section{Funding}

This manuscript is based on and contains results from a chapter of the author's doctoral dissertation, a project supervised by Bernt Øksendal and funded by the Research Council of Norway [grant number 125669].

\section{ORCID}

Nils Framstad (D) http://orcid.org/0000-0003-4529-4578

\section{References}

[1] R. Agnew, Counter-examples to an assertion concerning the normal distribution and a new stochastic price fluctuation model, Rev. Econ. Stud. 38 (1971), pp. 381-383.

[2] D. Applebaum, Lévy Processes and Stochastic Calculus, Vol. 93, Cambridge Studies in Advanced Mathematics, Cambridge University Press, Cambridge, 2004.

[3] G.B. Asheim, Intergenerational equity, Ann. Rev. Econ. 2 (2010), pp. 197-222.

[4] G.B. Asheim, and S. Zuber, A complete and strongly anonymous leximin relation on infinite streams, Soc. Choice Welfare 41 (2013), pp. 819-834. 
[5] L. Belkacem, J.L. Véhel, and C. Walter, CAPM, risk and portfolio selection in " $\alpha$-stable markets", Fractals 08 (2000), pp. 99-115.

[6] K. Borch, A note on uncertainty and indifference curves, Rev. Econ. Stud. 36 (1969), pp. 1-4.

[7] B.O. Bradley and M.S. Taqqu, Financial risk and heavy tails, in Handbook of Heavy Tailed Distributions in Finance, S.T. Rachev, ed., Elsevier, Amsterdam, 2003, pp. 35-103.

[8] S. Cambanis, S. Huang, and G. Simons, On the theory of elliptically contoured distributions, J. Multivariate Anal. 11 (1981), pp. 368-385.

[9] D. Cass, and J.E. Stiglitz, The structure of investor preferences and asset returns, and separability in portfolio allocation: A contribution to the pure theory of mutual funds, J. Econ. Theory 2 (1970), pp. 122-160.

[10] G. Chamberlain, A characterization of the distributions that imply mean-variance utility functions, J. Econ. Theory 29 (1983), pp. 185-201.

[11] B. de Finetti, Il problema dei "pieni" [The problem of full-risk insurances], Giorn. Ist. Ital. Attuari 11 (1940), pp. 1-88. Available at http://www.brunodefinetti.it/Opere/ Il20problema20dei20pieni.pdf

[12] N. Dokuchaev, Mutual fund theorem for continuous time markets with random coefficients, Theory Decis. 76 (2014), pp. 179-199.

[13] R.M. Dudley, Wiener functionals as Itô integrals, Ann. Probab. 5 (1977), pp. 140-141.

[14] E.F. Fama, Portfolio analysis in a stable Paretian market, Manage. Sci. 11 (1965), pp. 404-419.

[15] E.F. Fama, Risk, return, and equilibrium, J. Political Econ. 79 (1971), pp. 30-55.

[16] M.S. Feldstein, Mean-variance analysis in the theory of liquidity preference and portfolio selection, Rev. Econ. Stud 36 (1969), pp. 5-12.

[17] N.C. Framstad, Coherent portfolio separation-inherent systemic risk?, Int. J. Theor. Appl. Finance 7 (2004), pp. 909-917.

[18] N.C. Framstad, On Portfolio Separation in the Merton Problem with Bankruptcy or Default, in Proceedings of the International Conference on Stochastic Analysis and Applications, Sergio Albeverio, Anne Boutet de Monvel, and Habib Ouerdiane, eds., Kluwer Academic Publishers, Dordrecht, 2004, pp. 249-265. Available at http://doi.org/10.1007/978-1-4020-2468-9.

[19] N.C. Framstad, Portfolio separation properties of the skew-elliptical distributions, with generalizations, Statist. Probab. Lett. 81 (2011), pp. 1862-1866.

[20] N.C. Framstad, Portfolio theory for $\alpha$-symmetric and pseudo-isotropic distributions: $k$-fund separation and the CAPM, J. Probab. Stat. 2015 (2015), 235452, 11 p. doi:10.1155/2015/235452.

[21] B. Gamrowski and S. Rachev, A testable version of the Pareto-stable CAPM, Math. Comput. Model. 29 (1999), pp. 61-81.

[22] E.D. Giorgi, T. Hens, and J. Mayer, A note on reward-risk portfolio selection and two-fund separation, Finance Res. Lett. 8 (2011), pp. 52-58.

[23] P. Guasoni and S. Robertson, Static fund separation of long-term investments, Math. Finance. 25 (2015), pp. 789-826.

[24] A.K. Gupta, T.T. Nguyen, and W.B. Zeng, Conditions for stability of laws with all projections stable, Sankhyā: Indian J. Stat., Ser. A (1961-2002) 56 (1994), pp. 438-443.

[25] A.K. Gupta, T. Varga, and T. Bodnar, Elliptically Contoured Models in Statistics and Portfolio Theory 2nd ed., Springer, New York, 2013.

[26] P. Hall, A Comedy of Errors: The canonical form for a stable characteristic function, Bull. London Math. Soc. 13 (1981), pp. 23-27.

[27] X.D. He, and X.Y. Zhou, Portfolio choice via quantiles, Math. Finance 21 (2011), pp. 203-231.

[28] A.W. Janicki, I. Popova, P.H. Ritchken, and P.H. Ritchken, Option pricing bounds in an $\alpha$-stable security market, Comm. Stat. Stoch. Models 13 (1997), pp. 817-839.

[29] T. Kamae, and U. Krengel, Stochastic partial ordering, Ann. Probab. 6 (1979), pp. 1044-1049.

[30] T. Kamae, U. Krengel, and G.L. O’Brien, Stochastic inequalities on partially ordered spaces, Ann. Probab. 5 (1977), pp. 899-912.

[31] A. Khanna and M. Kulldorff, A generalization of the mutual fund theorem, Finance Stoch. 3 (1999), pp. 167-185.

[32] B.B. Mandelbrot, Fractals and Scaling in Finance, Springer-Verlag, New York, 1997. 
[33] D.J. Marcus, Nonstable laws with all projections stable, Z. Wahrsch. Verw. Gebiete 64 (1983), pp. 139-156.

[34] H. Markowitz, Portfolio selection, J. Finance 7 (1952), pp. 77-91.

[35] H. Markowitz, de Finetti scoops Markowitz, J. Invest. Manage. 4 (2006), pp. 3-18.

[36] J.H. McCulloch, Measuring tail thickness to estimate the stable index $\alpha$ : A critique, J. Bus. Econ. Stat. 15 (1997), pp. 74-81.

[37] J.H. McCulloch, Skew-stable investment opportunity set, 1999. Available at http://www.econ. ohio-state.edu/jhm/ios.html.

[38] R.C. Merton, Optimum consumption and portfolio rules in a continuous-time model, J. Econ. Theory 3 (1971), pp. 373-413.

[39] R.C. Merton, Erratum: "Optimum consumption and portfolio rules in a continuous-time model", J. Econ. Theory. 3 (1971), pp. 373-413; J. Econ. Theory, 6 (1973), pp. 213-214.

[40] R.C. Merton, Continuous-time Finance, Revised ed., Blackwell, Oxford, 1992.

[41] J.K. Misiewicz, Infinite divisibility of sub-stable processes. I. Geometry of sub-spaces of $L_{\alpha}$-space, Stoch. Process. Appl. 56 (1995), pp. 101-116.

[42] J.K. Misiewicz, Infinite divisibility of sub-stable processes. II. Logarithm of probability measure, J. Math. Sci. 81 (1996), pp. 2970-2979.

[43] S. Mittnik and S.T. Rachev, Stable Paretian Models in Finance, Wiley, New York, 2000.

[44] J.P. Nolan, Stable distributions - Models for heavy tailed data, book in progress, 2014. Available at http://academic2.american.edu/ jpnolan.

[45] B. Øksendal, Stochastic Differential Equations. An Introduction with Applications. 5th ed., Universitext, Springer-Verlag, Berlin, 1998.

[46] S. Ortobelli, I. Huber, S.T. Rachev, and E.S. Schwartz, Portfolio choice theory with non-Gaussian distributed returns, in Handbook of Heavy Tailed Distributions in Finance, S.T. Rachev, ed., Elsevier, Amsterdam, 2003, pp. 547-594.

[47] S. Ortobelli, I. Huber, and E. Schwartz, Portfolio selection with stable distributed returns, Math. Methods Oper. Res. 55 (2002), pp. 265-300.

[48] L.P. Østerdal, The mass transfer approach to multivariate discrete first order stochastic dominance: Direct proof and implications, J. Math. Econ. 46 (2010), pp. 1222-1228.

[49] J. Owen, and R. Rabinovitch, On the class of elliptical distributions and their applications to the theory of portfolio choice, J. Finance 38 (1983), pp. 745-752.

[50] F. Pressacco, and P. Serafini, The origins of the mean-variance approach in finance: revisiting de Finetti 65 years later, Decis. Econ. Finance 30 (2007), pp. 19-49.

[51] P.E. Protter, Stochastic Integration and Differential Equations, Stochastic Modelling and Applied Probability 2nd ed., Vol. 21, Springer-Verlag, Berlin, 2005. Version 2.1, Corrected third printing.

[52] S.T. Rachev, ed. Handbook of Heavy Tailed Distributions in Finance. Elsevier, Amsterdam, 2003.

[53] S.A. Ross, Mutual fund separation in financial theory - The separating distributions, J. Econ. Theory 17 (1978), pp. 254-286.

[54] A.D. Roy, Safety first and the holding of assets, Econometrica 20 (1952), pp. 431-449.

[55] G. Samorodnitsky and M.S. Taqqu, Stable non-Gaussian Random Processes. Stochastic Models with Infinite Variance, Chapman \& Hall, New York, 1994.

[56] P.A. Samuelson, Efficient portfolio selection for Pareto-Lévy investments, J. Financ. Quant. Anal. 2 (1967), pp. 107-122.

[57] P.A. Samuelson, General proof that diversification pays, J. Financ. Quant. Anal. 2 (1967), pp. $1-13$.

[58] W. Schachermayer, M. Sîrbu, and E. Taflin, In which financial markets do mutual fund theorems hold true?, Finance Stoch. 13 (2009), pp. 49-77.

[59] I.J. Schoenberg, Metric spaces and completely monotone functions, Ann. Math. (2). 39 (1938), pp. 811-841. Available at http://www.ams.org/mathscinet/search/journaldoc.html?cn=Ann of_Math_2.

[60] I.J. Schoenberg, Metric spaces and positive definite functions, Trans. Am. Math. Soc. 44 (1938), pp. 522-536. 
[61] S.P. Sethi and M. Taksar, A note on R. C. Merton's: "Optimum consumption and portfolio rules in a continuous-time model”, J. Econ. Theory. 46 (1988), pp. 395-401. Available at http://dx. doi.org/10.1016/0022-0531(88)90138-X.

[62] J. Tobin, Liquidity preference as behavior toward risk, Rev. Econ. Stud. 25 (1958), pp. 65-86.

[63] L. Barone, Bruno de Finetti Centenary Conference, Paper prepared for the "Bruno de finetti and the case of the critical line's last segment", Accademia dei Lincei, Rome, 2006. Available at http://www.brunodefinetti.it/Bibliografia/Last\%20Segment.pdf.

[64] L. Barone, Bruno de finetti, the problem of "full-risk insurances", J. Invest. Manage. 4 (2006), pp. 19-43.

\section{Appendix 1. Proofs}

Proof of Lemma 3.11: If not for the set constraint on $\boldsymbol{v}_{\varpi}\left(T_{d}^{+}\right)$, existence would follow from the construction of the integral, along with the requirement of Definition 3.6 that $v$ be integrable, which by dominated convergence for the stochastic integral ([51, Theorem IV.32]) ensures the convergence claimed. In order to accommodate the constraint, modify an approximation $\tilde{\boldsymbol{v}}_{\varpi}$ into one satisfying the set constraint by replacing $\tilde{\boldsymbol{v}}_{\varpi}(t)$ by the closest $\boldsymbol{v}_{\varpi}(t) \in \mathbb{V}_{T_{d}}$ (this distance tends to zero a.s. by the last part of Assumption 3.2(e)), though truncate to zero when this leads to Euclidean norm $\geq A$, and again apply dominated convergence.

For the separation claim, consider first the possible range of $s=\left\|x^{*}\right\| /\|x\|$ such that $\boldsymbol{x}^{\top} \boldsymbol{Z} \sim$ $\frac{s\|\boldsymbol{x}\|}{\left\|\boldsymbol{x}^{*}\right\|} \boldsymbol{x}^{* \top} \boldsymbol{Z}$ holds for a pair $\boldsymbol{x}$ and $\boldsymbol{x}^{*}$ both $\in \mathbb{V} \backslash\{\mathbf{0}\}$. If $s$ is not bounded away from 0 , we would violate Assumption 3.2(f) of no arbitrage nor redundant opportunities (here we use the shape of $\mathbb{V}$, so that there are no "asymptotic» arbitrages nor redundant opportunities as $\|\boldsymbol{x}\|$ grows). Interchanging rôles of $\boldsymbol{x}$ and $\boldsymbol{x}^{*}$, we also have an upper bound. We shall use this to bound the ratio $\left\|\boldsymbol{v}^{*}\right\| /\|\boldsymbol{v}\|$ in the following to a compact, for convergence.

Fix an arbitrary admissible strategy $(C, D, \boldsymbol{v})$ approximated by $\left(C_{\varpi}, D_{\varpi}, \boldsymbol{v}_{\varpi}\right)$. By assumption, there exists a $\left(C_{\varpi}^{*}, D_{\varpi}^{*}, \boldsymbol{v}_{\varpi}^{*}\right)$ satisfying $(4)$ with $\boldsymbol{v}^{*}$ spanned by $k$ risky funds. As the funds can be taken to belong to the (compact!) unit sphere, we can pass to a convergent subsequence as the partition refines - and so also for the unit vector $\boldsymbol{v}_{\varpi}^{*} /\left\|\boldsymbol{v}_{\varpi}^{*}\right\|$ and by the above argument, $\boldsymbol{v}^{*}$. (We should consider separately the set on which $\boldsymbol{v}_{\varpi}^{*}$ and hence $\boldsymbol{v}$ vanishes.) In the limit in probability (hence in distribution), positivity (or zeroness) of increments of $K$ and/or $D$ is preserved. Hence the limiting strategy is both admissible (by Definition 3.6 because $(C, D, v)$ is), and preferred to $(C, D, v)$.

Proof of Theorem 3.12: Let $\boldsymbol{f}(t)$ maximize $\boldsymbol{x}^{\top} \boldsymbol{\mu}$ subject to $\boldsymbol{x}^{\top} \Gamma \Gamma^{\top} \boldsymbol{x}$ and $\boldsymbol{x} \in \mathbb{V}_{t}$, noting that since $\mathbb{V}_{t}$ is closed and radial, this problem has a solution; if the solution is not unique, choose one so that $t \mapsto f$ is a Borel function. Consider an arbitrary strategy $(C, D, v)$. Fix a bounded time-interval where we discretize to a partition $\varpi$, where the strategy $\left(C_{\varpi}, D_{\varpi}, \boldsymbol{v}_{\varpi}\right)$ is $T_{d}$-measurable between $T_{d}$ and $T_{d+1}$. By Assumption 3.2(c) replace this $\mathfrak{H}_{T_{d}}^{(Y, C)} \vee \mathfrak{S}^{L\left(T_{d}\right)}$-measurable random variable by a variable of $\mathfrak{H}_{T_{d}}^{(Y, C)}$-conditionally same distribution; that is, a function of wealth-consumption history, and if applicable, randomization. Define for each $T_{d}$ in the partition, $Q_{\boldsymbol{v}_{\varpi}}\left(T_{d}\right)=\left(\boldsymbol{v}_{\varpi}{ }^{\top} \Gamma \Gamma^{\top}\right.$ $\left.\boldsymbol{v}_{\varpi}\right)^{1 / 2}$, also specified this way. Put $\boldsymbol{v}_{\varpi}^{*}\left(T_{d}\right)=Q_{\boldsymbol{v}_{\varpi}}\left(T_{d}\right) \boldsymbol{f}\left(T_{d}\right) . T_{d}$-conditionally, $\boldsymbol{v}_{\varpi}^{* T} \Gamma \mathrm{d} \boldsymbol{Z}$ and $\boldsymbol{v}_{\varpi}{ }^{\top} \Gamma \mathrm{d} \boldsymbol{Z}$ are on $\left(T_{d}, T_{d+1}\right]$ both Brownian motions on the same $T_{d}$-measurable time-change (indeed, $\sim Q \mathrm{~d} Z_{0}$ for some standard Brownian $Z_{0}$ by Lévy's characterization, see Øksendal [45, Section 8.5]) and thus have the same $T_{d}$-conditional law.

Assume first free disposal, and consider a strategy where we use the portfolio $\boldsymbol{v}_{\varpi}^{*}$ and dispose of wealth at (nonnegative and measurable!) rate $\mathrm{d} D=\left(\boldsymbol{v}_{\varpi}^{*}-\boldsymbol{v}_{\varpi}\right)^{\top} \boldsymbol{\mu} \mathrm{d} t$ on $\left(T_{d}, T_{d+1}\right]$. Thus, we keep actual consumption the same (and $T_{d}$-measurable), so that we can replace $\left\{Y(t)-Y\left(T_{d}^{+}\right)\right\}_{t \in\left(T_{d}, T_{d+1}\right]}$ by (the $T_{d}$-conditionally iid) $\left\{Y^{*}(t)-Y^{*}\left(T_{d}^{+}\right)\right\}_{t \in\left(T_{d}, T_{d+1}\right]}$, defining $Y^{*}=Y$ up to $T_{d}$ and thus using the same consumption - almost surely at $T_{d}^{+}$. We then have identical law up to $T_{d+1}$. From then on, replace $(Y, C)$ and whatever randomization the agent must have employed, by $\left(Y, C^{*}\right)$ and the appropriate $\boldsymbol{L}$ in the functional form of the consumption-portfolio decisions - without changing the $T_{d+1}$-conditional law, since the driving noise is Lévy. Using the strong Markov property and the independent increments of Lévy processes, the wealth-consumption processes coincide in law on $\mathbb{R}$. Now, reformulate by inserting for feedback functions to express all processes and controls as 
$(t, \omega)$-functions. We can then can drop the assumption of free disposal: if it does not hold, improve by consuming the excess $\mathrm{d} K:=\left(\boldsymbol{v}_{\varpi}^{*}-\boldsymbol{v}_{\varpi}\right)^{\top} \boldsymbol{\mu} \mathrm{d} t$ (measurable!) rather than disposing of it. The result is still admissible by Definition 3.6 provided the original strategy is.

Finally, pass from partitions to the limit in probability (hence distribution, hence preserving the equivalence in law) using Lemma 3.11.

Notice that invoking the Lévy characterization is a bit of an overkill: we do not need that the processes are conditionally Brownian, only that they coincide in (conditional) law on the interval. This will be used in later proofs.

Proof of Lemma 4.1: Decompose the Lévy measure into $\lambda=\sum_{k=0}^{\infty} \lambda_{l}$ where $\lambda_{0}=\lambda 1_{\{\|\boldsymbol{\delta}\|>1\}}$ and each $\lambda_{l}=\lambda 1_{\left\{\|\delta\| \in\left(2^{-l}, 2^{1-l}\right]\right\}}$ are finite - and by assumption, $\lambda_{l}$ isotropic. Consider the jump part of $\ln \mathrm{E}\left[\exp \left(\mathrm{i} \boldsymbol{\xi}^{\top} \boldsymbol{Z}(1)\right)\right]$; i.e. by the Lévy-Khintchine representation (e.g., [2, ch. 1])

$$
\int\left[e^{\mathrm{i} \boldsymbol{\xi}^{\top} \boldsymbol{\delta}}-1-\mathrm{i} \boldsymbol{\xi}^{\top} \boldsymbol{\delta} 1_{\|\boldsymbol{\delta}\| \leq 1}\right] \sum_{l=0}^{\infty} \lambda_{l}(\mathrm{~d} \boldsymbol{\delta})=\sum_{l=0}^{\infty}\{\underbrace{\int e^{\mathrm{i} \boldsymbol{\xi}^{\top} \boldsymbol{\delta}} \lambda_{l}(\mathrm{~d} \boldsymbol{\delta})-\lambda_{l}\left(\mathbb{R}^{m}\right)}_{=\left[h_{l}(\|\boldsymbol{\xi}\|)-1\right] \cdot \lambda_{l}\left(\mathbb{R}^{m}\right)}\}
$$

The truncated compensation term on the left-hand side cancels by the symmetry of each $\lambda_{l}$. The underbrace form follows by identifying the integral as the "characteristic function» of the finite isotropic measure $\lambda_{l}$, and using the representation (7). So the (convergent!) sum over $l$ thus depends on $\xi$ only through its Euclidean norm. With the Gaussian component assumed to be a scaling $\sigma$ of a standard Brownian motion, we have an additional term $\exp \left(-\sigma^{2}\|\xi\|^{2} / 2\right)$, still a function of only $\|\xi\|$.

Proof of Theorem 4.3: The very same proof as in Theorem 3.12 will suffice for part (a), although the « $Z_{0}$ » of the proof of Theorem 3.12 is not Brownian (which, as remarked, it need not be). That is, the distribution of the increments is determined by the drift and the quadratic form $Q_{v}^{2}=\boldsymbol{v}^{\top} \boldsymbol{E} \boldsymbol{v}$ : As long as $Q_{\boldsymbol{v}^{*}(t)}(t)=Q_{\boldsymbol{v}(t)}(t)$ we have $\boldsymbol{v}^{* \top} \Gamma \mathrm{d} \boldsymbol{Z} \sim \boldsymbol{v}^{\top} \Gamma \mathrm{d} \boldsymbol{Z}$, and a dominating strategy is found by choosing $\boldsymbol{v}^{*}$ such that $\left(\boldsymbol{v}^{*}(t)-\boldsymbol{v}(t)\right)^{\top} \boldsymbol{\mu}(t) \geq 0$ for each $t$. In the radial case (a), this amounts to $\boldsymbol{v}^{*}=\|\boldsymbol{v}\| \boldsymbol{f}$ where $\boldsymbol{f}$ maximizes for $Q=1$, and if non-unique maximum, selected so that $t \mapsto \boldsymbol{f}$ is Borel.

Also in the other cases, the recipe from Theorem 3.12 will take care of the transition to continuous time. This leaves us with solving the static problems (6) for each $t$. Let us focus on case (b) without risk-free opportunity. We solve for each $T_{d}$ the following Lagrange problem, with $Y_{\varpi}\left(T_{d}^{+}\right)$for $Y$ :

$$
\max \boldsymbol{x}^{\top} \boldsymbol{\mu} \quad \text { subject to } \quad \boldsymbol{x}^{\top} \boldsymbol{\Xi} \boldsymbol{x}=Q^{2}, \quad \boldsymbol{x}^{\top} \mathbf{1}=Y,
$$

though only for those $Q$ for which there is a feasible point. E.g., when $Q^{2}=Y^{2}$ there is only one, namely the "minimum variance portfolio» $Y \boldsymbol{f}_{1}=Y \operatorname{argmin}_{\boldsymbol{x}^{\top} \mathbf{1}=1} \boldsymbol{x}^{\top} \boldsymbol{E} \boldsymbol{x}$ and this is the only point where the constraint qualification for the Lagrange problem may possibly fail. The Lagrange firstorder condition reads $\boldsymbol{\mu}=2 \ell_{0} \boldsymbol{\Xi} \boldsymbol{x}+\ell \mathbf{1}$. $\Xi$ is assumed invertible, so as long as $\ell_{0} \neq 0$, we have $\boldsymbol{x}$ spanned by $\Xi^{-1} \boldsymbol{\mu}$ and $\boldsymbol{f}_{1}=\Xi^{-1} \mathbf{1} /\left(\mathbf{1}^{\top} \Xi^{-1} \mathbf{1}\right)$. Should $\ell_{0}$ vanish, we cannot use $\Xi^{-1} \boldsymbol{\mu}$ for $\boldsymbol{f}_{2}$, as $\boldsymbol{\mu}$ must be $=\bar{\mu}_{0} \mathbf{1}$; then the maximization degenerates, and one can choose the second fund to be any vector $\boldsymbol{f}_{2}$ such that $\boldsymbol{f}_{2}^{\top} \boldsymbol{E} \boldsymbol{f}_{1}=0$ and $\boldsymbol{f}_{2}^{\top} \boldsymbol{E} \boldsymbol{f}_{2}=1$. So, the funds can be chosen invariant over $Y$ and $Q$. Case (c) works like the latter except that we cannot merely choose any $\boldsymbol{f}_{2}$. If $\boldsymbol{f}_{2}^{\top} \boldsymbol{E} \boldsymbol{f}_{2}$ is bounded, choose the maximizer (since $\mathbb{V}$ is convex, $\boldsymbol{f}_{1}+\boldsymbol{f}_{2} \cdot q /\left(\boldsymbol{f}_{2}^{\top} \boldsymbol{\Xi} \boldsymbol{f}_{2}\right)$ will be admissible for all $q \in[0,1])$; if unbounded, choose one in a linear direction from $f_{1}$ where arbitrary high volatility is attained.

Part (d) is shown analogously, just with more constraints, leading to more funds. For part (e), we adapt part (d): for a given strategy $(C, D, v)$, the agent chooses $\boldsymbol{v}(t)^{\top} \mathbf{1}=b$, and thus we impose the constraint $\boldsymbol{x}^{\top} \mathbf{1}=b$, and maximize the drift after cost, which now becomes $\boldsymbol{x}^{\top} \boldsymbol{\mu}-b \hat{\rho}(b)$, the latter term not affecting the maximization.

Proof of Theorem 4.5: Consider an approximating sequence converging to the Itô integral (10) - i.e. a sequence of partitions $\varpi$ on which we have not only an approximating strategy, but also 
approximate $M(t)$ with steps $M\left(T_{d}\right)$ on $\left(T_{d}, T_{d+1}\right]$. In this approximation, the opportunity set at time $T_{d+1}$ is determined by $(Y, M)$, and - in the approximation - this is bivariate Gaussian with $\operatorname{drift}\left(\boldsymbol{v}_{\varpi}\left(T_{d}^{+}\right)^{\top} \boldsymbol{\mu}\left(T_{d}, M\left(T_{d}\right)\right), \bar{\mu}\left(T_{d}, M\left(T_{d}\right)\right)\right)^{\top}$ and covariance as stated:

$$
\left(\begin{array}{c}
\boldsymbol{v}_{\varpi \varpi}^{\top} \Gamma \\
\boldsymbol{\gamma}^{\top}
\end{array}\right)\left(\Gamma^{\top} \boldsymbol{v}_{\varpi} \boldsymbol{\gamma}\right)=\left(\begin{array}{cc}
\boldsymbol{v}_{\varpi}^{\top} \Gamma \Gamma^{\top} \boldsymbol{v}_{\varpi} & \boldsymbol{v}_{\varpi \varpi}^{\top} \Gamma \boldsymbol{\gamma} \\
\boldsymbol{v}_{\varpi}^{\top} \Gamma \boldsymbol{\gamma} & \boldsymbol{\gamma}^{\top} \boldsymbol{\gamma}
\end{array}\right)
$$

(times $\mathrm{d} t$ ); here, both $\boldsymbol{v}_{\varpi}$ and coefficients are evaluated at $\left(T_{d}^{+}, M\left(T_{d}^{+}\right)\right)$. Thus, to improve over $\boldsymbol{v}_{\varpi}\left(T_{d}^{+}\right)$we choose the $\boldsymbol{x}$ that maximizes $\boldsymbol{x}^{\top} \boldsymbol{\mu}$ subject to the constraints $\boldsymbol{x}^{\top} \Gamma \Gamma^{\top} \boldsymbol{x}=\boldsymbol{v}_{\varpi}\left(T_{d}^{+}\right)^{\top} \Gamma \Gamma^{\top}$ $\boldsymbol{v}_{\varpi}\left(T_{d}^{+}\right)$and (9) augmented with yet an additional linear constraint, namely $\boldsymbol{x}^{\top} \Gamma \boldsymbol{\gamma}=\boldsymbol{v}_{\varpi}\left(T_{d}^{+}\right)^{\top} \Gamma \boldsymbol{\gamma}$. The solution to this static problem follows as in Theorem 4.3. We thus have a dominating strategy on the partition. Refining the partition as in Lemma 3.11, gives the result in continuous time.

Proof of Theorem 5.1 and Corollary 5.2: As mentioned, part (a) is [20, Theorem 9], and follows like if we put $\beta=0$ in part (b).

The closedness claimed in part (b), follows by bounded convergence. Let $x \in \mathbb{H}$ : then (14) implies $\int_{ \pm \mathbb{H} \cap \mathbb{S}}\left|\boldsymbol{x}^{\top} \boldsymbol{s}\right|^{\alpha} \mathrm{d} \varkappa=\int_{\mathbb{H} \cap \mathbb{S}}\left(\boldsymbol{x}^{\top} \boldsymbol{s}\right)^{\alpha} \mathrm{d} \bar{\varkappa} \cdot(1 \pm \beta) / 2$, so that $\sigma_{\boldsymbol{x}}^{\alpha}=\int\left(\boldsymbol{x}^{\top} \boldsymbol{s}\right)^{\alpha} \mathrm{d} \bar{\varkappa}$ and $\beta_{\boldsymbol{x}}=\beta$.

For the stochastic dominance part, let first $\alpha \neq 1$ : then $\sigma_{x} f^{\top} \boldsymbol{X} \sim \boldsymbol{x}^{\top} \boldsymbol{X}+\sigma_{\boldsymbol{x}}\left(\boldsymbol{f}-\boldsymbol{x} / \sigma_{\boldsymbol{x}}\right)^{\top} \boldsymbol{\mu}$ because they have the same drift and scale (by construction) and the same skewness (by assumption).

Let $\alpha=1$. Location now has an additional term; insert $\sigma_{x} \cdot\left(\boldsymbol{x} / \sigma_{\boldsymbol{x}}\right)$ to rewrite $\mu_{\boldsymbol{x}}$ into

$$
\sigma_{x}\left[\frac{x^{\top}}{\sigma_{x}} \boldsymbol{\mu}-\frac{2}{\pi} \int \frac{\boldsymbol{x}^{\top}}{\sigma_{x}} \boldsymbol{s} \ln \left|\frac{\boldsymbol{x}^{\top}}{\sigma_{x}} \boldsymbol{s}\right| \mathrm{d} \varkappa\right]-\sigma_{x} \frac{2}{\pi} \ln \sigma_{x} \frac{\int \boldsymbol{x}^{\top} \boldsymbol{s} \mathrm{d} \varkappa}{\sigma_{x}}=\sigma_{x}\left[\mu_{x / \sigma_{x}}-\frac{2}{\pi} \beta_{x} \ln \sigma_{x}\right]
$$

Since $\beta_{x}$ is constantly $=\beta$ on the radial $\mathbb{V}$, the solution of $\max \mu_{x}$ subject to $\mathbb{V}$ and to scale $=Q$, is again $Q f$ where $\boldsymbol{f}$ solves the problem for $\sigma_{x}=Q=1$.

To show the Corollary, notice again that formula (14) yields skewness of $\beta$ and scale of $\sigma_{v}=$ $\int\left(\boldsymbol{v}^{\top} \boldsymbol{s}\right)^{\alpha} \mathrm{d} \bar{\varkappa}$, valid on $\mathbb{V}$. For $\alpha=1$ without risk-free opportunity, $\sigma_{v}$ then equals $\bar{\zeta} Y$. Thus Theorem 5.1 part (b) applies outright with the same $f$ : removing the risk-free opportunity merely restricts the choice of $\sigma_{v}$ to a singleton.

For the transition to continuous time in part (c) of the theorem, copy the proofs of Theorem 4.3 part (a) or Theorem 3.12 with minor modifications as follows. With the stable distributions of (b), consider an approximating step strategy, note that on $\left(T_{d}, T_{d+1}\right]$ we have both $\boldsymbol{v}_{\bar{\omega}}^{\top}{ }^{\top} \Delta \boldsymbol{Z}$ and $\boldsymbol{v}_{\varpi}^{* \top} \triangle \boldsymbol{Z}$ being $T_{d}$-conditionally $\alpha$-stable with the same skewness and scale; as $\left(\boldsymbol{v}^{*}-\boldsymbol{v}\right)^{\top} \boldsymbol{\mu} \geq 0$, we generate an improvement. The pseudo-isotropic distributions of (a) work likewise. Pass to continuous time by refining partitions. 